\title{
The molecular gas reservoir of 6 low-metallicity galaxies from the Herschel Dwarf Galaxy Survey
}

\section{A ground-based follow-up survey of $\mathrm{CO}(1-0), \mathrm{CO}(2-1)$, and $\mathrm{CO}(3-2)$}

\author{
D. Cormier ${ }^{1}$, S. C. Madden ${ }^{2}$, V. Lebouteiller ${ }^{2}$, S. Hony ${ }^{3}$, S. Aalto ${ }^{4}$, F. Costagliola ${ }^{4,5}$, A. Hughes ${ }^{3}$, A. Rémy-Ruyer ${ }^{2}$, \\ N. Abel ${ }^{6}$, E. Bayet ${ }^{7}$, F. Bigiel ${ }^{1}$, J. M. Cannon ${ }^{8}$, R. J. Cumming ${ }^{9}$, M. Galametz ${ }^{10}$, F. Galliano ${ }^{2}$, S. Viti ${ }^{11}$, and R. Wu ${ }^{2}$ \\ ${ }^{1}$ Institut für theoretische Astrophysik, Zentrum für Astronomie der Universität Heidelberg, Albert-Ueberle Str. 2, \\ 69120 Heidelberg, Germany \\ e-mail: diane.cormier@zah.uni-heidelberg.de \\ ${ }^{2}$ Laboratoire AIM, CEA/DSM - CNRS - Université Paris Diderot, Irfu/Service d'Astrophysique, CEA Saclay, \\ 91191 Gif-sur-Yvette, France \\ 3 Max-Planck-Institute for Astronomy, Königstuhl 17, 69117 Heidelberg, Germany \\ ${ }^{4}$ Department of Earth and Space Sciences, Chalmers University of Technology, Onsala Space Observatory, 43992 Onsala, Sweden \\ 5 Instituto de Astrofísica de Andalucía, Glorieta de la Astronomía s/n, 18008 Granada, Spain \\ ${ }^{6}$ University of Cincinnati, Clermont College, Batavia OH 45103, USA \\ 7 Sub-Dept. of Astrophysics, Dept. of Physics at University of Oxford, Denys Wilkinson Building, Keble Road, Oxford OX1 3RH, \\ UK \\ 8 Department of Physics \& Astronomy, Macalester College, 1600 Grand Avenue, Saint Paul MN 55105, USA \\ 9 Onsala Space Observatory, Chalmers University of Technology, 43992 Onsala, Sweden \\ 10 Institute of Astronomy, University of Cambridge, Madingley Road, Cambridge CB3 OHA, UK \\ 11 Department of Physics and Astronomy, University College London, Gower Street, London WC1E 6BT, UK
}

Received 17 June 2013 / Accepted 17 November 2013

\section{ABSTRACT}

\begin{abstract}
Context. Observations of nearby starburst and spiral galaxies have revealed that molecular gas is the driver of star formation. However, some nearby low-metallicity dwarf galaxies are actively forming stars, but CO, the most common tracer of this reservoir, is faint, leaving us with a puzzle about how star formation proceeds in these environments.

Aims. We aim to quantify the molecular gas reservoir in a subset of 6 galaxies from the Herschel Dwarf Galaxy Survey with newly acquired $\mathrm{CO}$ data and to link this reservoir to the observed star formation activity.

Methods. We present $\mathrm{CO}(1-0), \mathrm{CO}(2-1)$, and $\mathrm{CO}(3-2)$ observations obtained at the ATNF Mopra 22-m, APEX, and IRAM 30-m telescopes, as well as [C II] $157 \mu \mathrm{m}$ and [O I] $63 \mu \mathrm{m}$ observations obtained with the Herschel/PACS spectrometer in the 6 low-metallicity dwarf galaxies: Haro 11, Mrk 1089, Mrk 930, NGC 4861, NGC 625, and UM 311. We derived their molecular gas masses from several methods, including using the CO-to- $\mathrm{H}_{2}$ conversion factor $X_{\mathrm{CO}}$ (both Galactic and metallicity-scaled values) and dust measurements. The molecular and atomic gas reservoirs were compared to the star formation activity. We also constrained the physical conditions of the molecular clouds using the non-LTE code RADEX and the spectral synthesis code Cloudy.

Results. We detect $\mathrm{CO}$ in 5 of the 6 galaxies, including first detections in Haro $11\left(Z \sim 0.4 Z_{\odot}\right)$, Mrk $930\left(0.2 Z_{\odot}\right)$, and UM 311 $\left(0.5 Z_{\odot}\right)$, but $\mathrm{CO}$ remains undetected in NGC $4861\left(0.2 Z_{\odot}\right)$. The $\mathrm{CO}$ luminosities are low, while [C II] is bright in these galaxies, resulting in $[\mathrm{C} \mathrm{II}] / \mathrm{CO}(1-0) \geq 10000$. Our dwarf galaxies are in relatively good agreement with the Schmidt-Kennicutt relation for total gas. They show short molecular depletion timescales, even when considering metallicity-scaled $X_{\mathrm{CO}}$ factors. Those galaxies are dominated by their H I gas, except Haro 11, which has high star formation efficiency and is dominated by ionized and molecular gas. We determine the mass of each ISM phase in Haro 11 using Cloudy and estimate an equivalent $X_{\mathrm{CO}}$ factor that is 10 times higher than the Galactic value. Overall, our results confirm the emerging picture that $\mathrm{CO}$ suffers from significant selective photodissociation in low-metallicity dwarf galaxies.
\end{abstract}

Key words. galaxies: ISM - galaxies: dwarf - ISM: molecules - infrared: galaxies - radio lines: galaxies

\section{Introduction}

On galactic scales, the star formation rate is observed to correlate with the total (molecular and atomic) gas reservoir, following the empirical Schmidt-Kennicutt law (e.g., Schmidt 1959; Kennicutt 1998):

$\Sigma_{\mathrm{SFR}} \propto\left(\Sigma_{\mathrm{gas}}\right)^{n}$, with $n \simeq 1.4$,

where $\Sigma_{\mathrm{SFR}}$ is the star formation rate surface density, and $\Sigma_{\text {gas }}$ the gas surface density. There is evidence that the star formation law in galaxies is mostly regulated by the molecular gas rather than the total gas content and that the timescale for converting molecular gas into stars is to first order constant for disk galaxies and around $\tau_{\text {dep }} \sim 2$ Gyr (Bigiel et al. 2008, 2011; Leroy et al. 2008; Genzel et al. 2012). Leroy et al. (2005) analyzed the star formation law in non-interacting dwarf galaxies of the northern hemisphere with metallicities $12+\log (\mathrm{O} / \mathrm{H}) \geq 8.0^{1}$.

1 The metallicity $12+\log (\mathrm{O} / \mathrm{H})$ is denoted by $\mathrm{O} / \mathrm{H}$ throughout the paper. 
They find that the center of dwarf galaxies and more massive spiral galaxies follow the same relationship between molecular gas, measured by $\mathrm{CO}(1-0)$, and the star formation rate (SFR), measured by the radio continuum, with a power-law index $n \simeq$ 1.2-1.3.

The tight correlation between star formation and molecular gas emission results from the conditions required for molecules to be abundant. High density is a prerequisite for star formation. To be protected against photodissociation, molecules also require a dense and shielded environment, where $\mathrm{CO}$ acts as a main coolant of the gas. At low metallicities, this correlation may not hold since other lines - particularly atomic fine-structure lines such as the [C II] $157 \mu \mathrm{m}$ line - can also cool the gas efficiently enough to allow stars to form (Krumholz et al. 2011; Glover \& Clark 2012). The formation of $\mathrm{H}_{2}$ on grain surfaces is also affected in these environments. At extremely low metallicities, below $1 / 100 Z_{\odot}{ }^{2}$, Krumholz (2012) demonstrates that the timescale for forming molecules is longer than the thermal and free-fall timescales. As a consequence, star formation may occur in the cold atomic phase before the medium becomes fully molecular (see also Glover \& Clark 2012).

On the observational side, many low-metallicity dwarf galaxies $\left(1 / 40 Z_{\odot} \leq Z \leq 1 / 2 Z_{\odot}\right)$ are forming stars but show little observed molecular gas as traced by $\mathrm{CO}$ emission, making them outliers in the Schmidt-Kennicutt relation (e.g., Galametz et al. 2009; Schruba et al. 2012). How star formation occurs in these environments is poorly known. Such a discrepancy with the Schmidt-Kennicutt relation may imply either higher star formation efficiencies (SFE) than in normal galaxies or larger total gas reservoirs than measured by $\mathrm{CO}$, as favored by recent studies (e.g., Schruba et al. 2012; Glover \& Clark 2012).

Most of the molecular gas in galaxies is in the form of cold $\mathrm{H}_{2}$, which is not directly traceable due to the lack of low-energy transitions (no dipole moment), leaving the second most abundant molecule, $\mathrm{CO}$, as the most common molecular gas tracer (see Bolatto et al. 2013 for a review on the CO-to- $\mathrm{H}_{2}$ conversion factor). $\mathrm{CO}$, however, is difficult to detect in low-metallicity dwarf galaxies. Does this imply that dwarf galaxies truly contain little molecular gas or that $\mathrm{CO}$ becomes a poor tracer of $\mathrm{H}_{2}$ in low-metallicity ISM? The dearth of detectable CO in dwarf galaxies can be a direct result of the chemical evolution (from lower $\mathrm{C}$ and $\mathrm{O}$ abundances) or else be due to the structure and conditions of the ISM. Modeling of photodissociation regions (PDRs) demonstrates the profound effects of the low metallicity on the gas temperatures, which often result in an enhanced $C O$ emission from higher- $J$ transitions, and on the structure of the PDR envelope (Röllig et al. 2006). While $\mathrm{H}_{2}$ is self-shielded from the UV photons and can survive in the PDR, CO survival depends on the dust shielding. $\mathrm{CO}$ is therefore more easily prone to photodestruction, especially in low-metallicity star-forming galaxies, which have both a hard radiation field and a low dustto-gas mass ratio, leaving only small filling factor molecular clumps, which are difficult to detect with single-dish antennas due to severe beam dilution effects (e.g., Taylor et al. 1998; Leroy et al. 2011; Schruba et al. 2012). This molecular gas not traced by $\mathrm{CO}$ has been referred to as the "dark gas" (Wolfire et al. 2010; Grenier et al. 2005).

The full molecular gas reservoir in dwarf galaxies should, therefore, include the molecular gas traced by $\mathrm{CO}$ and the CO-dark gas that emits brightly in other emission lines. PDR tracers, such as [O I] and [C II], which usually arise from the surface layer of $\mathrm{CO}$ clouds and hence co-exist with the CO-dark

\footnotetext{
2 The solar metallicity is taken as $(\mathrm{O} / \mathrm{H})_{\odot}=8.7$ (Asplund et al. 2009).
}

molecular gas (Poglitsch et al. 1995; Madden et al. 1997; Glover et al. 2010), can trace the CO-dark gas. The [C II] $157 \mu \mathrm{m}$ farinfrared (FIR) fine-structure line is one of the most important coolants of the ISM (Tielens \& Hollenbach 1985; Wolfire et al. 1995), and it was first used in Madden et al. (1997) to quantify the total molecular gas reservoir in a dwarf galaxy. New evidence of the presence of a significant reservoir of CO-dark molecular gas, on the order of 10 to 100 times what is inferred by CO (Madden 2000), is suggested by the exceptionally high [C II]-to-CO ratios found in the Dwarf Galaxy Survey (DGS; Madden et al. 2013; Cormier et al. 2010), a Herschel Key Program that has observed 50 nearby low-metallicity dwarf galaxies in the FIR/submillimeter (submm) dust bands and the FIR fine-structure lines of the ionized and neutral gas, including [C II] $157 \mu \mathrm{m}$ and [O I] $63 \mu \mathrm{m}$.

In this paper, we present new $\mathrm{CO}$ observations of six dwarf galaxies from the DGS, Haro 11, Mrk 930, Mrk 1089, NGC 4861, NGC 625, and UM 311, with metallicities ranging from $1 / 6$ to $1 / 2 Z_{\odot}$ (Table 1 ). Section 2 describes the observations and data reduction of the $\mathrm{CO}(1-0), \mathrm{CO}(2-1)$, and $\mathrm{CO}(3-2)$ data sets, as well as the [C II] $157 \mu \mathrm{m}$ and [O I] $63 \mu \mathrm{m}$ lines from Herschel and the warm $\mathrm{H}_{2}$ lines from Spitzer. We quantify the physical conditions of the molecular phase in Sect. 4, using empirical diagnostics, the non-local thermal equilibrium (non-LTE) code RADEX, and excitation diagrams for the warm $\mathrm{H}_{2}$ gas. In particular, we focus our analysis on comparing the cold and warm molecular gas reservoirs that are inferred from several methods ( $X_{\mathrm{CO}}$ conversion factor, dust, etc.). In addition, we apply a full radiative transfer modeling to the low-metallicity galaxy Haro 11 as a case study in Sect. 5, in order to characterize properties of the CO-dark gas in the PDR. We then discuss our results in the context of the overall star formation activity in these galaxies, and investigate how the estimated amount of molecular gas relates to other galaxy properties (atomic reservoir, SFR, etc.; Sect. 6). Throughout the paper, the quoted molecular gas masses refer to $\mathrm{H}_{2}$ masses, except in Sect. 6 where the masses are multiplied by a factor 1.36 to include helium in the total gas reservoir.

\section{Surveying the neutral gas in low-metallicity galaxies: [C II], [O I], $\mathrm{H}_{2}$, and $\mathrm{CO}$ data}

\subsection{Target selection}

Owing to the intrinsically faint $\mathrm{CO}$ and instrumental sensitivity limitations, studies of $\mathrm{CO}$ in low-metallicity environments have essentially focused on Local Group galaxies, such as the Magellanic Clouds, IC 10, NGC 6822, or M 33. The proximity of the Magellanic Clouds, for example, has allowed for detailed studies of molecular clouds, where the MAGMA survey achieves $11 \mathrm{pc}$ resolution with Mopra (Wong et al. 2011), and ALMA resolves even sub-pc structures (Indebetouw et al. 2013). Those Local Group galaxies do, however, probe a limited range of physical conditions. Outside of the Local Group, CO is detected in tidal dwarf galaxies (e.g., Braine et al. 2001) and Magellanic-type spirals and irregular galaxies (Albrecht et al. 2004; Leroy et al. 2005, 2009), but detections are sparse in blue compact dwarf (BCD) galaxies (e.g., Israel 2005). From the DGS, only the galaxies NGC 1569, NGC $6822\left(1 / 5-1 / 6 Z_{\odot}\right)$, Haro 2, Haro 3, II Zw 40, IC 10, NGC 4214, NGC 5253 (1/3 Z $\left.{ }_{\odot}\right)$, and $\mathrm{He} 2-10\left(1 / 2 Z_{\odot}\right)$ have been observed in more than one CO transition (Sage et al. 1992; Kobulnicky et al. 1995; Taylor et al. 1998; Meier et al. 2001; Bayet et al. 2004; Israel 2005). To date, $\mathrm{CO}$ has only been detected in dwarf galaxies with 
Table 1. General properties of the DGS dwarf subsample.

\begin{tabular}{|c|c|c|c|c|c|c|c|c|c|c|}
\hline \multirow{2}{*}{$\begin{array}{l}\text { Galaxy } \\
\text { Haro } 11\end{array}$} & \multicolumn{2}{|c|}{$\begin{array}{c}\text { Coordinates } \\
(\mathrm{J} 2000) \\
\end{array}$} & \multirow{2}{*}{$\begin{array}{c}\begin{array}{c}\text { Dist. } \\
(\mathrm{Mpc})\end{array} \\
92\end{array}$} & \multirow{2}{*}{$\begin{array}{c}\text { Optical size } \\
0.4^{\prime} \times 0.5^{\prime} \\
(11 \mathrm{kpc} \times 13 \mathrm{kpc})\end{array}$} & \multirow{2}{*}{$\begin{array}{c}\begin{array}{c}\text { Metallicity } \\
\mathrm{O} / \mathrm{H}\end{array} \\
8.20^{(a)}\end{array}$} & \multirow{2}{*}{$\begin{array}{c}\begin{array}{c}M_{\mathrm{B}} \\
(\mathrm{mag})\end{array} \\
-20.6\end{array}$} & \multirow{2}{*}{$\begin{array}{c}\begin{array}{c}M_{\mathrm{H} \mathrm{I}} \\
\left(10^{9} M_{\odot}\right)\end{array} \\
0.5^{(b)}\end{array}$} & \multirow{2}{*}{$\begin{array}{c}\begin{array}{c}L_{\mathrm{TIR}} \\
\left(10^{9} L_{\odot}\right)\end{array} \\
185\end{array}$} & \multirow{2}{*}{$\begin{array}{c}\begin{array}{c}L_{\mathrm{H} \alpha} \\
\left(10^{7} L_{\odot}\right)\end{array} \\
92\end{array}$} & \multirow{2}{*}{$\begin{array}{c}\begin{array}{c}L_{\mathrm{FUV}} \\
\left(10^{9} L_{\odot}\right)\end{array} \\
22\end{array}$} \\
\hline & $00 \mathrm{~h} 36^{\prime} 52.5^{\prime \prime}$ & $-33^{\circ} 33^{\prime} 19^{\prime \prime}$ & & & & & & & & \\
\hline Mrk 1089 & $05 \mathrm{~h} 01^{\prime} 37.8^{\prime \prime}$ & $-04^{\circ} 15^{\prime} 28^{\prime \prime}$ & 57 & $\begin{array}{c}0.6^{\prime} \times 0.2^{\prime} \\
(10 \mathrm{kpc} \times 3 \mathrm{kpc})\end{array}$ & 8.10 & -20.5 & 24 & 34.3 & 23 & - \\
\hline Mrk 930 & $23 \mathrm{~h} 31^{\prime} 58.3^{\prime \prime}$ & $+28^{\circ} 56^{\prime} 50^{\prime \prime}$ & 78 & $\begin{array}{c}0.4^{\prime} \times 0.4^{\prime} \\
(9 \mathrm{kpc} \times 9 \mathrm{kpc})\end{array}$ & 8.03 & -19.5 & 3.0 & 18.8 & 23 & - \\
\hline NGC 4861 & $12 \mathrm{~h} 59^{\prime} 02.3^{\prime \prime}$ & $+34^{\circ} 51^{\prime} 34^{\prime \prime}$ & 7.5 & $\begin{array}{c}4.0^{\prime} \times 1.5^{\prime} \\
(9 \mathrm{kpc} \times 3 \mathrm{kpc})\end{array}$ & 7.89 & -16.8 & 0.48 & 0.323 & 0.04 & 0.46 \\
\hline NGC 625 & $01 \mathrm{~h} 35^{\prime} 04.6^{\prime \prime}$ & $-41^{\circ} 26^{\prime} 10^{\prime \prime}$ & 3.9 & $\begin{array}{c}5.8^{\prime} \times 1.9^{\prime} \\
(7 \mathrm{kpc} \times 2 \mathrm{kpc})\end{array}$ & 8.22 & -16.2 & 0.13 & 0.280 & 0.18 & 0.11 \\
\hline UM 311 & $01 \mathrm{~h} 15^{\prime} 34.0^{\prime \prime}$ & $-00^{\circ} 51^{\prime} 32^{\prime \prime}$ & 24 & $\begin{array}{c}0.1^{\prime} \times 0.1^{\prime} \\
(0.7 \mathrm{kpc} \times 0.7 \mathrm{kpc})\end{array}$ & 8.38 & -19.2 & 3.0 & 5.18 & 0.48 & - \\
\hline
\end{tabular}

Notes. Coordinates and optical sizes are taken from the NASA/IPAC Extragalactic Database. The size of UM 311 is that of the individual H II galaxy, but the complex that we consider is larger $\left(\sim 3^{\prime} \times 2^{\prime}\right)$. Distances, metallicities, $M_{\mathrm{B}}$, and H I masses are from (Madden et al. 2013, see references therein). For Mrk 930, $M_{\mathrm{B}}$ is from Markarian et al. (1989). Luminosities and masses are scaled to the quoted distances. $L_{\mathrm{TIR}}$ is the total infrared (TIR) luminosity measured by integrating SEDs from 8 to $1000 \mu \mathrm{m}$, from Rémy-Ruyer et al. (in prep.). ${ }^{(a)}$ For Haro 11 , we keep the metallicity used in Cormier et al. (2012). ${ }^{(b)}$ From MacHattie et al. (2014) and S. Pardy (priv. comm.), see details in Sect. 2.

References. For $L_{\mathrm{H} \alpha}$ : Haro 11: Östlin et al. (1999); UM 311: Terlevich et al. (1991); Mrk 930: Adamo et al. (2011b); NGC 4861: Schmitt et al. (2006); Mrk 1089: Iglesias-Paramo \& Vilchez (1997); and NGC 625: Kennicutt et al. (2008). For $L_{\mathrm{Fuv}}$ : Haro 11: Grimes et al. (2007); NGC 4861: Gil de Paz et al. (2007); and NGC 625: Lee et al. (2011).

metallicities $\geq 1 / 6 Z_{\odot}$, with the exception of the close-by galaxy WLM (Elmegreen et al. 2013), which has a metallicity of $\sim 1 / 8 Z_{\odot}$.

To complement the existing $\mathrm{CO}$ data for the galaxies in the DGS, we have observed $\mathrm{CO}$ in six additional low-metallicity galaxies of the DGS: Haro 11, Mrk 930, Mrk 1089, NGC 625, NGC 4861, and UM 311. The general properties of these galaxies are summarized in Table 1 . They are all subsolar metallicity and larger than a kpc in size. In particular, NGC 625 and NGC 4861 are classified as SBm galaxies and have lower (optical and IR) luminosities than the other galaxies. This target selection is essentially limited by instrument sensitivity: our sample consists of the brightest - in the infrared - galaxies of the DGS that lack CO data. Mrk 930, NGC 4861, and UM 311 have no $\mathrm{CO}$ observations published at all. $\mathrm{CO}(1-0)$ observations were attempted in Haro 11 by Bergvall et al. (2000) without success. We observed Haro 11 and UM 311 in the three transitions $\mathrm{CO}(1-0), \mathrm{CO}(2-1)$, and $\mathrm{CO}(3-2)$; and Mrk 930 and the giant $\mathrm{H}$ II region I Zw 49 in NGC 4861 in $\mathrm{CO}(1-0)$ and $\mathrm{CO}(2-1)$. Data of CO(1-0) already existed for Mrk 1089 (Leon et al. 1998), and of $\mathrm{CO}(2-1)$ and $\mathrm{CO}(3-2)$ for NGC 625 (partial coverage, P.I. Cannon). Thus for these two galaxies we observed the CO transitions that were lacking. NGC 625 was observed in $\mathrm{CO}(1-0)$ with $4 \times 1$ pointings, while the other galaxies are more compact and required only single pointings. The $\mathrm{CO}$ beams are overlaid on HST images in Fig. 1.

Haro 11 - also known as ESO 350-IG 038, is a blue compact galaxy (BCG) located at $92 \mathrm{Mpc}$, with metallicity $Z \sim 1 / 3 Z_{\odot}$ (James et al. 2013). Haro 11 hosts extreme starburst conditions with star formation rate $\sim 25 M_{\odot} \mathrm{yr}^{-1}$ (Grimes et al. 2007). It was previously observed in $\mathrm{HI}$ and $\mathrm{CO}$ but not detected (Bergvall et al. 2000). Interestingly, $\mathrm{H}$ I has recently been detected in absorption by MacHattie et al. (2014), yielding a mass of $M(\mathrm{HI})$ between $3 \times 10^{8}$ and $10^{9} M_{\odot}$, and in emission with the GBT, yielding an $\mathrm{H}$ I mass of $4.7 \times 10^{8} M_{\odot}$ (S. Pardy, priv. comm.). Both H I masses agree with each other and are higher than the upper limit from Bergvall et al. (2000), thus we use the average value of $5 \times 10^{8} M_{\odot}$.

Mrk 1089 - is part of the Hickson Group 31 (Hickson 1982). It is an irregular starburst (HCG31 C) at $57 \mathrm{Mpc}$, with metallicity $\sim 1 / 3 Z_{\odot}, S F R \simeq 16 M_{\odot} \mathrm{yr}^{-1}$ (Hopkins et al. 2002), and in interaction with the galaxy NGC 1741 (HCG31 A). Since the two galaxies are not clearly separated in the mid-infrared (MIR)-FIR bands, we refer to the group as Mrk 1089, as in Galametz et al. (2009). HCG31 C dominates the emission in these bands and is the only one detected in CO (Leon et al. 1998).

Mrk 930 - is a blue compact galaxy located at $78 \mathrm{Mpc}$ with metallicity $\sim 1 / 5 Z_{\odot}$. It is also classified as a Wolf-Rayet (WR) galaxy (Izotov \& Thuan 1998), with WR signatures indicating that it is undergoing a young burst episode. It exhibits an intricate morphology with several sites of active star formation. Its star cluster age distribution peaks at $4 \mathrm{Myr}$ and it has an SFR of $\sim 6 M_{\odot} \mathrm{yr}^{-1}$ (Adamo et al. 2011b).

$N G C 4861$ - is an irregular galaxy with a cometary shape, located at $7.5 \mathrm{Mpc}$, and with metallicity $Z \sim 1 / 6 Z_{\odot}$. It is classified as an SB(s)m galaxy in the de Vaucouleurs et al. (1991) catalog, but shows no evidence of a spiral structure (Wilcots et al. 1996). It hosts several knots of star formation, dominated by the southwestern region IZw 49 (where our CO observations point), as seen in $\mathrm{H} \alpha$ (Van Eymeren et al. 2009). The $\mathrm{H} \alpha$ and H I distributions coincide in the center, but the $\mathrm{H}$ I extends to form a larger envelope. Its $\mathrm{SFR}$ is $\sim 0.05 M_{\odot} \mathrm{yr}^{-1}$.

$N G C 625$ - is an irregular elongated dwarf galaxy in the Sculptor Group located at $4 \mathrm{Mpc}$ and with metallicity $\sim 1 / 3 Z_{\odot}$. It is undergoing an extended starburst $\geq 50 \mathrm{Myr}$, with $S F R \simeq$ $0.05 M_{\odot} \mathrm{yr}^{-1}$ (Skillman et al. 2003), which resulted in an outflow visible in H I (Cannon et al. 2004, 2005). It is detected in $\mathrm{CO}(2-1)$ and $\mathrm{CO}(3-2)$ toward the main dust concentration, and shows MIR $\mathrm{H}_{2}$ lines in its Spitzer/IRS spectrum (Sect. 2.6).

UM 311 - is one of a group of three compact $\mathrm{H}$ II galaxies at a distance of $23 \mathrm{Mpc}$ and with metallicity $\sim 1 / 2 Z_{\odot}$, located between the pair of spiral galaxies NGC 450 and UGC 807. It is bright in $\mathrm{H} \alpha$ (Guseva et al. 1998) and H I (Smoker et al. 2000), with $S F R \simeq 1 M_{\odot} \mathrm{yr}^{-1}$ (Hopkins et al. 2002). The individual sources of the group are separated by $\sim 15^{\prime \prime}$ and are not resolved in the FIR photometry, although UM 311 dominates the emission. When referring to UM 311, we encompass the full complex (the three H II galaxies and the two spirals). 

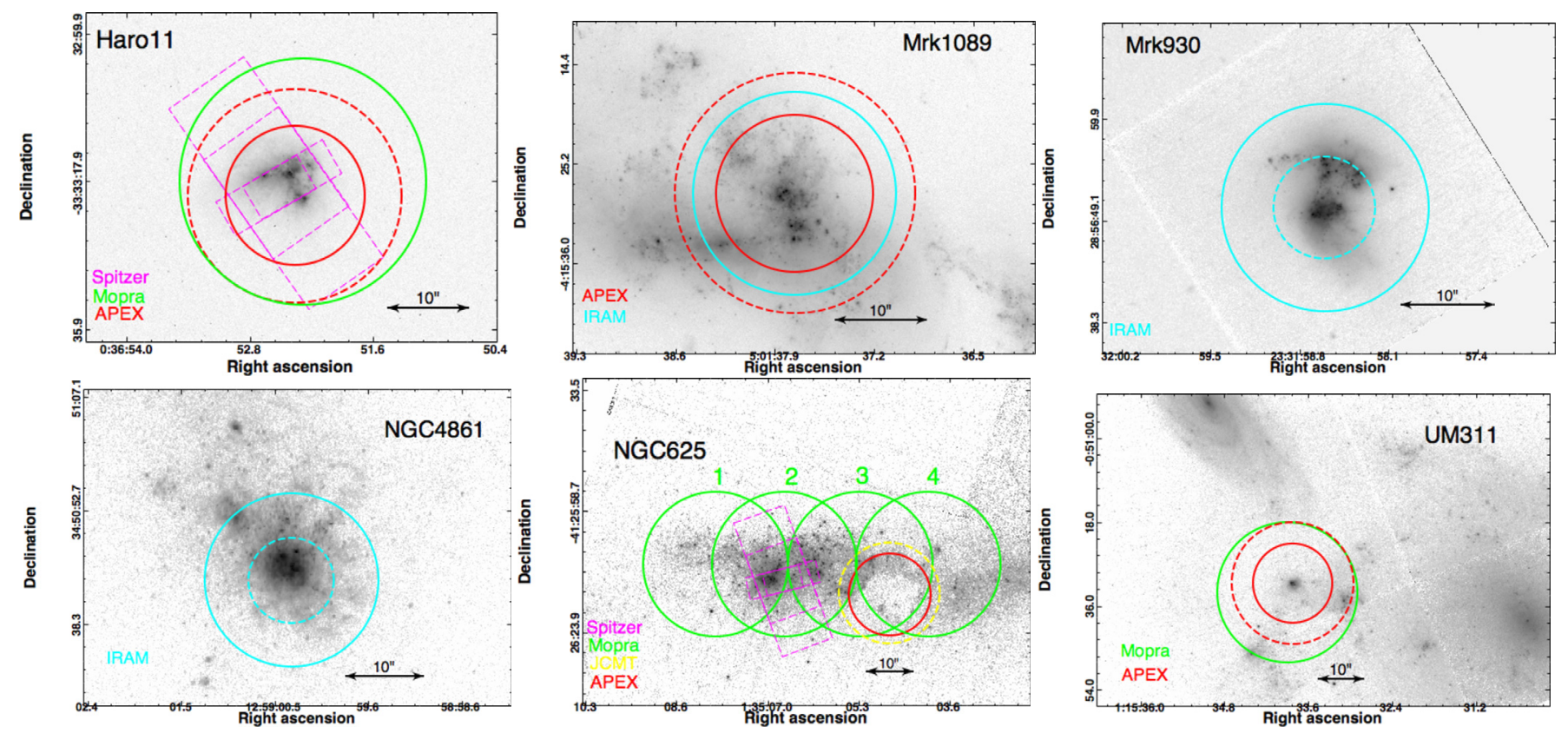

Fig. 1. HST broad band images of the 6 galaxies surveyed in CO (WFPC2 or ACS: F300 for NGC 625, F814 for UM 311 , and F606 for the others), downloaded from the Hubble Legacy Archive (http://hla.stsci . edu/). The Mopra beam is shown in green (CO(1-0): $\left.30^{\prime \prime}\right)$, APEX in red (CO (2-1): 26", $\left.\mathrm{CO}(3-2): 17^{\prime \prime}\right)$, IRAM in blue (CO(1-0): 21", $\mathrm{CO}(2-1)$, dashed: $\left.11^{\prime \prime}\right)$, and the JCMT in yellow (CO(2-1), dashed: 22") for NGC 625. The Spitzer/IRS footprints are also shown in magenta for Haro 11 and NGC 625.

\subsection{Mopra data: $\mathrm{CO}(1-0)$ in southern hemisphere targets}

We observed the $\mathrm{CO}(1-0)$ line in Haro 11, NGC 625, and UM 311 with the ATNF Mopra 22-m telescope as part of the program M596, from September 13 to 18, 2011. Observations consisted of a single pointing for Haro 11 and UM 311, and of a $4 \times 1$ pointed map with a half-beam step size for NGC 625 . The size of the beam is $\sim 30^{\prime \prime}$ at $115 \mathrm{GHz}$. The position-switching observing mode consists of a pattern of four minutes: OFF1ON-ON-OFF2, where OFF1 and OFF2 are two different reference positions in the sky taken $\geq 1.5^{\prime}$ away from the source, to remove the contribution from the sky. We used the 3-mm band receiver and the backend MOPS in wideband mode, scanning a total bandwidth of $8.2 \mathrm{GHz}$ with spectral resolution $0.25 \mathrm{MHz}$ and central frequency $109.8 \mathrm{GHz}$ for Haro $11,111.5 \mathrm{GHz}$ for UM 311, and $112.0 \mathrm{GHz}$ for NGC 625. The above-atmosphere system temperature was estimated every $20 \mathrm{~min}$ using an ambient (hot) load. Between hot load measurements, fluctuations in the system temperature are monitored using a noise diode. The total $(\mathrm{ON}+\mathrm{OFF})$ integration times were $14.1 \mathrm{~h}, 10.3 \mathrm{~h}$, and $3.0 \mathrm{~h}$ for Haro 11, UM 311, and NGC 625 respectively. The average system temperatures were 265,375 , and 370 kelvins. We used Orion KL to monitor the absolute flux calibration and the following pointing sources: R Hor, R Dor, S Scl, R Apr, and o Ceti.

We used our own interactive data language (IDL)-written script for the data reduction. We first create a quotient spectrum taking the average of the two reference position spectra before and after the on-source spectrum. The equation for the quotient spectrum $Q$ with continuum removal is $Q=T_{\mathrm{OFF}} \times(\mathrm{ON} / \mathrm{OFF})-$ $\mathrm{T}_{\mathrm{ON}}$, where $\mathrm{ON}$ and $\mathrm{OFF}$ are the on-source and off-source spectra. We then fit a spline function to remove the large scale variations and shift the signal to a median level of zero. The data are calibrated using a main beam efficiency of $\eta_{\mathrm{mb}}=0.42 \pm 0.02$ (Ladd et al. 2005). The pointing errors are estimated to be $\leq 3.5^{\prime \prime}$.

With Mopra, we detect CO(1-0) in NGC 625, in beam position 3 (counting from east to west, see Fig. 1), and a possible detection in position 2. It is surprising that no line is detected in position 4 , since both positions 3 and 4 cover the area where the ancillary $\mathrm{CO}(2-1)$ and $\mathrm{CO}(3-2)$ observations were taken and the lines detected. This could indicate that the $\mathrm{CO}$ emission detected in position 3 comes from a region not covered by position 4 or the ancillary data, and the emission from the ancillary data is not seen by Mopra because of its limited sensitivity. We can therefore only compute an upper limit on the flux in the area covered by the ancillary data to be able to compare the $\mathrm{CO}(1-0)$ to the other transitions. The spectra from all positions are displayed in Fig. 2, and the corresponding line parameters are reported in Table 2. For the total $\mathrm{CO}$ emission, we consider the average flux of all four positions and an average beam size taken as the geometric area covered by the four pointings.

$\mathrm{CO}(1-0)$ is not detected with Mopra in Haro 11 or in UM 311. Their spectra are strongly affected by $\sim 30 \mathrm{MHz}$ baseline ripples, caused by standing waves in the receiversubreflector cavity. To counter this effect, we selected two different reference positions and applied a defocusing procedure while observing, but the standing waves were still present in our data. Since they change with frequency, it is difficult to model or filter them. As a first step, we identify the spectra affected by the ripples by eye. Ten per cent show ripples with strong amplitude and standard deviation $>110 \mathrm{mK}$, and $20 \%$ with ripples of lower amplitude but clear wave shapes. Flagging out these spectra, we then rebinned the data to a spectral resolution of $10 \mathrm{~km} \mathrm{~s}^{-1}$, and did a sigma-weighted average to obtain a final spectrum, with no additional correction. The $\mathrm{CO}(1-0)$ lines remain undetected in Haro 11 and UM 311.

To improve our final rms further, we used a defringing tool based on a routine originally developed for removing fringes from ISO SWS spectroscopic data (Kester et al. 2003). The routine was modified for our purpose and tuned to specifically search for a maximum of ten significant fringes in the frequency domain of the fringes seen in the deep Mopra data. It is applied to all individual spectra. After subtracting the ripples, the noise level is greatly reduced. Again, the final spectra, shown 

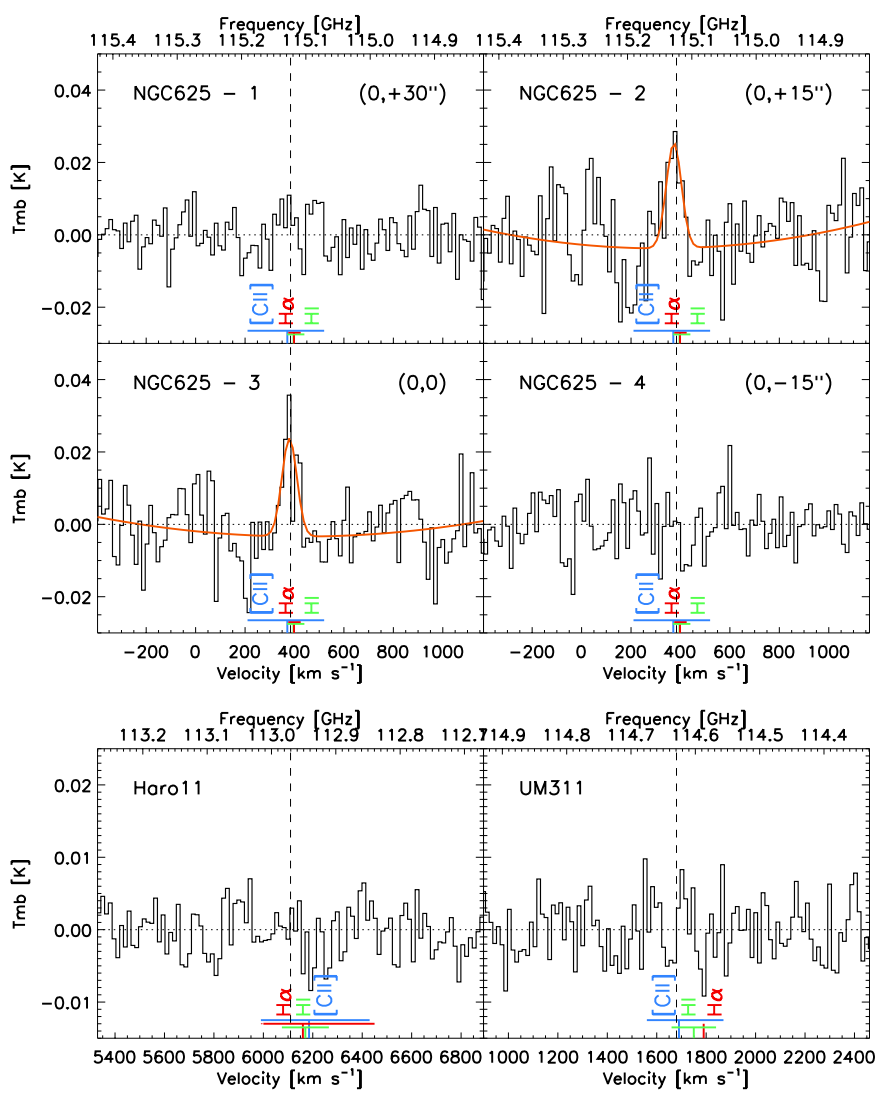

Fig. 2. Mopra spectra $\left(T_{\mathrm{mb}}\right)$ of the $\mathrm{CO}(1-0)$ line at $115 \mathrm{GHz}$ in NGC 625 , Haro 11 , and UM 311 . The $(0,0)$ position in NGC 625 (pointing 3) has coordinates (RA, Dec) $=\left(01^{\mathrm{h}} 35^{\mathrm{m}} 05.46^{\mathrm{s}},-4^{\mathrm{d}} 26^{\mathrm{m}} 09.6^{\mathrm{s}}\right)$. The spectral resolution for the display is $15 \mathrm{~km} \mathrm{~s}^{-1}$, corresponding to $\sim 5.8 \mathrm{MHz}$. The orange curve shows the fit to the line when detected. The vertical dashed lines indicate the expected position of the line, based on the central velocity of the other $\mathrm{CO}$ transitions. Central velocities of [C II], $\mathrm{HI}$, and $\mathrm{H} \alpha$, and their dispersions are also indicated in blue, green, and red, respectively. All velocities are indicated in the local standard-of-rest (LSR) reference frame.

in Fig. 2, are obtained with a sigma-weighted average of all spectra. Bergvall et al. (2000) report an incredibly low limit of $L_{\mathrm{CO}(1-0)}<260 L_{\odot}$ in Haro 11 , but without giving details on their calculation. To be conservative, we prefer to use our upper limit throughout this paper.

\subsection{APEX data: $\mathrm{CO}(2-1)$ and $\mathrm{CO}(3-2)$ in southern hemisphere targets}

We observed the $\mathrm{CO}(2-1)$ and $\mathrm{CO}(3-2)$ lines in Haro 11, Mrk 1089, and UM 311 with the Swedish Heterodyne Facility Instrument (SHeFI) on the Atacama Pathfinder EXperiment (APEX). These observations are part of the program, ID 088.F9316A, and were performed from September 3 to 11, 2011. The beam size is $\sim 26^{\prime \prime}$ at $230 \mathrm{GHz}$ and $\sim 17^{\prime \prime}$ at $345 \mathrm{GHz}$. We made single pointings in $\mathrm{ON} / \mathrm{OFF}$ mode with the receivers APEX 1 and APEX 2 and backend XFFTS. The bandwidth covered is $2.5 \mathrm{GHz}$, with initial spectral resolution $88.5 \mathrm{kHz}$. Total integration times for each source (ON source + OFF source) were 82 and $120 \mathrm{~min}$ for Haro 11,79 and $140 \mathrm{~min}$ for UM 311, and 135 and $110 \mathrm{~min}$ for Mrk 1089 for the $\mathrm{CO}(2-1)$ and $\mathrm{CO}(3-2)$ lines, respectively. Calibration was done every ten minutes. The average system temperatures were 150, 248, 156, 214, 153, $208 \mathrm{~K}$. The following pointing and focus sources were used: PI1-GRU,
WX-PSC, O-CETI, R-LEP, TX-PSC, RAFGL3068, IK-TAU, $\mathrm{R}-\mathrm{SCL}$, Uranus. The pointing accuracy is typically $2^{\prime \prime}$.

We also present $\mathrm{CO}(3-2)$ observations of NGC 625, obtained between August 17 and 26, 2005 as part of the program 00.F-0007-2005 (P.I.: J.M. Cannon) and retrieved from the ESO archive. Observations consisted of a single pointing (Fig. 1) with the receiver APEX 2 and backend FFTS (decommissioned), which is composed of two $1 \mathrm{GHz}$ units achieving a spectral resolution of $122 \mathrm{kHz}$. The total integration time was $155 \mathrm{~min}$, and the average system temperature was $150 \mathrm{~K}$. The sources R-FOR and R-SCL were used for pointing, and Jupiter for focus.

The data reduction was performed with the GILDAS ${ }^{3}$ software CLASS. The spectra are all averaged together and smoothed to a resolution of $\sim 10-15 \mathrm{~km} \mathrm{~s}^{-1}\left(3 \mathrm{~km} \mathrm{~s}^{-1}\right.$ for NGC 625). They are converted to main beam brightness temperature, $T_{\mathrm{mb}}=T_{\mathrm{a}}^{*} / \eta_{\mathrm{mb}}$, with main beam efficiencies ${ }^{4}$ of 0.75 and 0.73 at 230 and $345 \mathrm{GHz}$ (with typical uncertainties of 5$10 \%$ ). We apply a third-order polynomial fit to the baseline and a Gaussian fit to the line to derive the line intensity. A sinusoid baseline subtraction is also applied to NGC 625. For total uncertainties, we add $20 \%$ to the uncertainties on the line fits. We detect the $\mathrm{CO}(2-1)$ and $\mathrm{CO}(3-2)$ lines in all observations (Fig. 3 and Table 2).

\subsection{IRAM observations: $\mathrm{CO}(1-0)$ and $\mathrm{CO}(2-1)$ in northern hemisphere targets}

We observed the $\mathrm{CO}(1-0)$ and $\mathrm{CO}(2-1)$ lines in Mrk 930 and NGC 4861 with the IRAM 30-m telescope from July 19 to 24, 2012. Observations were done in wobbler-switching mode, with a throw of $4^{\prime}$ for NGC 4861 and 1.5' for Mrk 930. They consisted of single pointings toward the peak of the [C II] emission. The beam sizes are $\sim 21^{\prime \prime}$ at $115 \mathrm{GHz}$ and $\sim 11^{\prime \prime}$ at $230 \mathrm{GHz}$. We used the EMIR receivers E090 and E230 simultaneously, with bandwidth coverage of $8 \mathrm{GHz}$ each, and with the backends WILMA ( $2 \mathrm{MHz}$ resolution) for $\mathrm{CO}(1-0)$ and FTS $(200 \mathrm{kHz}$ resolution) for $\mathrm{CO}(2-1)$. The total integration times (ON+OFF) were 455 and 407 min for NGC 4861 and 538 and 548 min for Mrk 930 for $\mathrm{CO}(1-0)$ and $\mathrm{CO}(2-1)$, respectively. The average system temperatures were 362 and $408 \mathrm{~K}$ for NGC 4861 and 190 and $355 \mathrm{~K}$ for Mrk 930. The temperature of the system was calculated every $15 \mathrm{~min}$, pointings were done every $1 \mathrm{~h}$ and focus every $2 \mathrm{~h}$ on average. For pointing and focus, we used the sources Mercury, Mars, Uranus 1039+811, 2251+158, 2234+282, 2200+420, NGC 7027, and for line calibrators: IRC+10216 and CRL2688. The pointing accuracy is estimated to be $\sim 3^{\prime \prime}$.

The 30-m data are also reduced with CLASS. Antenna temperatures are corrected for main beam efficiencies $B_{\text {eff }}{ }^{5}$ of 0.78 and 0.60 at 115 and $230 \mathrm{GHz}$, using $T_{\mathrm{mb}}=T_{\mathrm{a}}^{*} \times F_{\text {eff }} / B_{\text {eff }}$. We have a marginal detection of the $\mathrm{CO}(1-0)$ line in Mrk 930, while $\mathrm{CO}$ is not detected in the other observations (Fig. 4) with rms values $\sim 2 \mathrm{mK}$. For total uncertainties, we add $20 \%$ to the uncertainties on the line fits. The line parameters, intensities, and upper limits can be found in Table 2 .

\subsection{Herschel data: $[\mathrm{C} \mathrm{II}]$ and $[\mathrm{O} \mathrm{I}]$}

The [C II] $157 \mu \mathrm{m}$ and [O I] $63 \mu \mathrm{m}$ fine-structure cooling lines were observed in the six galaxies with the PACS spectrometer

\footnotetext{
3 http://wWW.iram.fr/IRAMFR/GILDAS

4 http://www .apex-telescope.org/telescope/efficiency/

5 http://www.iram.es/IRAMES/mainWiki/

Iram30mEfficiencies
} 
Table 2. CO line parameters and intensities.

\begin{tabular}{|c|c|c|c|c|c|c|c|c|c|}
\hline Galaxy & Line & Center & $\begin{array}{l}\theta_{\text {FWHM }} \\
\left({ }^{\prime \prime}\right)\end{array}$ & $\begin{array}{c}T_{\mathrm{mb}} \\
(\mathrm{mK})\end{array}$ & $\begin{array}{c}V \\
\left(\mathrm{~km} \mathrm{~s}^{-1}\right) \\
\end{array}$ & $\begin{array}{c}\Delta V \\
\left(\mathrm{~km} \mathrm{~s}^{-1}\right)\end{array}$ & $\begin{array}{c}I_{\mathrm{CO}} \\
\left(\mathrm{K} \mathrm{km} \mathrm{s}^{-1}\right)\end{array}$ & $\begin{array}{l}L_{\mathrm{CO}} \\
\left(L_{\odot}\right)\end{array}$ & Reference \\
\hline \multirow[t]{3}{*}{ Haro 11} & $\mathrm{CO}(1-0)$ & & $30^{(1)}$ & $<10.7^{(a)}$ & - & - & $<0.69$ & $<6.6 \times 10^{3}$ & this work \\
\hline & $\mathrm{CO}(2-1)$ & & $26^{(2)}$ & 8.63 & 6113 & 59 & $0.54 \pm 0.19$ & $3.2 \times 10^{4}$ & this work \\
\hline & $\mathrm{CO}(3-2)$ & & $17^{(2)}$ & 7.20 & 6103 & 63 & $0.48 \pm 0.17$ & $4.0 \times 10^{4}$ & this work \\
\hline \multirow[t]{3}{*}{ Mrk 1089} & $\mathrm{CO}(1-0)$ & & $21^{(3)}$ & 26.3 & 3987 & 148 & $2.56 \pm 0.50$ & $4.7 \times 10^{3}$ & Leon et al. (1998) \\
\hline & $\mathrm{CO}(2-1)$ & & 26 & 14.1 & 3990 & 111 & $1.67 \pm 0.40$ & $3.8 \times 10^{4}$ & this work \\
\hline & $\mathrm{CO}(3-2)$ & & 17 & 11.5 & 3976 & 95 & $1.16 \pm 0.32$ & $3.8 \times 10^{4}$ & this work \\
\hline \multirow[t]{2}{*}{ Mrk 930} & $\mathrm{CO}(1-0)$ & & 21 & 2.17 & 5491 & 62 & $0.14 \pm 0.07$ & $4.7 \times 10^{2}$ & this work \\
\hline & $\mathrm{CO}(2-1)$ & & $11^{(3)}$ & $<6.44$ & - & - & $<0.34$ & $<2.6 \times 10^{3}$ & this work \\
\hline \multirow[t]{3}{*}{ NGC 4861} & $\mathrm{CO}(1-0)$ & & 21 & $<6.51$ & - & - & $<0.35$ & $<1.1 \times 10^{1}$ & this work \\
\hline & $\mathrm{CO}(1-0)$ & & total $^{(b)}$ & - & - & - & $<1.23$ & $<3.9 \times 10^{1}$ & this work \\
\hline & $\mathrm{CO}(2-1)$ & & 11 & $<8.74$ & - & - & $<0.47$ & $<3.3 \times 10^{1}$ & this work \\
\hline \multirow[t]{8}{*}{ NGC 625} & $\mathrm{CO}(1-0)$ & $\left(0,+30^{\prime \prime}\right)$ & 30 & $<24.9$ & - & - & $<1.86$ & $<3.3 \times 10^{1}$ & this work \\
\hline & $\mathrm{CO}(1-0)$ & $\left(0,+15^{\prime \prime}\right)$ & 30 & 29.0 & 373 & 72 & $2.23 \pm 0.72$ & $4.0 \times 10^{1}$ & this work \\
\hline & $\mathrm{CO}(1-0)$ & $(0,0)$ & 30 & 27.1 & 379 & 71 & $2.05 \pm 0.63$ & $3.6 \times 10^{1}$ & this work \\
\hline & $\mathrm{CO}(1-0)$ & $\left(0,-15^{\prime \prime}\right)$ & 30 & $<21.6$ & - & - & $<1.61$ & $<2.9 \times 10^{1}$ & this work \\
\hline & $\mathrm{CO}(1-0)$ & & total $^{(b)}$ & 57.7 & 374 & 70 & $4.26 \pm 0.62$ & $7.5 \times 10^{1}$ & this work \\
\hline & $\mathrm{CO}(1-0)$ & $\operatorname{ref}^{(c)}$ & 30 & - & - & - & $<1.61^{(c)}$ & $<2.9 \times 10^{1}$ & this work \\
\hline & $\mathrm{CO}(2-1)$ & & $22^{(4)}$ & 58.0 & 390 & 27 & $1.64 \pm 0.40$ & $1.0 \times 10^{2}$ & Cannon (2004) \\
\hline & $\mathrm{CO}(3-2)$ & & 17 & 59.0 & 381 & 23 & $1.44 \pm 0.38$ & $2.2 \times 10^{2}$ & this work \\
\hline \multirow[t]{4}{*}{ UM 311} & $\mathrm{CO}(1-0)$ & & 30 & $<11.4$ & - & - & $<0.61$ & $<4.1 \times 10^{2}$ & this work \\
\hline & $\mathrm{CO}(1-0)$ & & total $^{(b)}$ & - & - & - & $<3.05$ & $<2.0 \times 10^{3}$ & this work \\
\hline & $\mathrm{CO}(2-1)$ & & 26 & 10.9 & 1678 & 41 & $0.47 \pm 0.15$ & $1.9 \times 10^{3}$ & this work \\
\hline & $\mathrm{CO}(3-2)$ & & 17 & 8.62 & 1686 & 44 & $0.40 \pm 0.14$ & $2.3 \times 10^{3}$ & this work \\
\hline
\end{tabular}

Notes. (1) ATNF/Mopra; (2) APEX/SHeFI; (3) IRAM/30-m; (4) JCMT/RxA.

(a) The upper limits are $3 \sigma .{ }^{(b)}$ Total emission for extended galaxies, estimated from the other Mopra pointings for NGC 625 and extrapolated from the $100 \mu \mathrm{m}$ continuum emission for NGC 4861 and UM 311 (see Sect. 3.2). ${ }^{(c)}$ Estimated CO(1-0) line intensity at the position covered by the $\mathrm{CO}(2-1)$ and $\mathrm{CO}(3-2)$ lines.

(Poglitsch et al. 2010) on board the Herschel Space Observatory (Pilbratt et al. 2010) as part of two Guaranteed Time Key Programs, the DGS (Madden et al. 2013) and SHINING (P.I. Sturm). The results of the full Herschel spectroscopic survey in dwarf galaxies is presented in Cormier et al. (in prep.). The PACS array is composed of $5 \times 5$ spatial pixels of size $9.4^{\prime \prime}$ each, covering a field-of-view of $47^{\prime \prime} \times 47^{\prime \prime}$. The [C II] observations consist of a $5 \times 2$ raster map for NGC 4861, and of $2 \times 2$ raster maps for the other galaxies. The [O I] observations consist of a $3 \times 3$ raster map for Mrk 1089, $2 \times 2$ raster maps for Haro 11 and UM 311, and single pointings for Mrk 930, NGC 625, NGC 4861. All observations were done in chop-nod mode with a chop throw of $6^{\prime}$ off the source (free of emission). The beam sizes are $\sim 9.5^{\prime \prime}$ and $11.5^{\prime \prime}$, and the spectral resolution $\sim 90 \mathrm{~km} \mathrm{~s}^{-1}$ and $240 \mathrm{~km} \mathrm{~s}^{-1}$ at $60 \mu \mathrm{m}$ and $160 \mu \mathrm{m}$, respectively (PACS Observer's Manual 2011).

The data were reduced with the Herschel Interactive Processing Environment User Release v9.1.0 (Ott 2010), using standard scripts of the PACS spectrometer pipeline, and analyzed with the in-house software PACSMAN v3.52 (Lebouteiller et al. 2012). For the line fitting, the signal from each spatial position of the PACS array is fit with a second-order polynomial plus Gaussian for the baseline and line. The rasters are combined by drizzling to produce final maps of $3^{\prime \prime}$ pixel size.

The line intensity maps of [C II] $157 \mu \mathrm{m}$ are displayed in Fig. 5. The [C II] emission is extended in NGC 625, NGC 4861, and UM 311, and marginally in Haro 11, Mrk 1089, and Mrk 930. The [O I] emission is relatively more compact. The signal-to-noise ratio is $\geq 15 \sigma$ on the peak of emission for both $[\mathrm{C}$ II $]$ and $[\mathrm{OI}]$. We measure line fluxes both from the area covered by the maps and in a circular aperture of size that of the
$\mathrm{CO}(1-0)$ beam (Table 3$)$. The uncertainties come from the noise and from fitting the spectra. Calibration errors add an additional $\sim 30 \%$ systematic uncertainty (Poglitsch et al. 2010) and thus dominate the total uncertainties.

\subsection{Spitzer/IRS data: warm $\mathrm{H}_{2}$}

All galaxies were observed with the Infrared Spectrograph (IRS; Houck et al. 2004) onboard the Spitzer Space Telescope (Werner et al. 2004), but $\mathrm{H}_{2}$ rotational lines are clearly seen in the spectra of Haro 11 and NGC 625 only, so we present the IRS data of these two galaxies. NGC 625 was observed on July 11, 2005 (P.I. Gehrz; AORKey 5051904) in staring mode with the highresolution modules SH $\left(\approx 10-20 \mu \mathrm{m}\right.$; slit size $\left.4.7^{\prime \prime} \times 11.3^{\prime \prime}\right)$ and LH $\left(\approx 19-37 \mu \mathrm{m}\right.$; slit size $\left.11.1^{\prime \prime} \times 22.3^{\prime \prime}\right)$. The observation points toward the coordinates $(\mathrm{RA}, \mathrm{Dec})=\left(1^{\mathrm{h}} 35^{\mathrm{m}} 06.8^{\mathrm{s}},-41^{\mathrm{d}} 26^{\mathrm{m}} 13^{\mathrm{s}}\right)$, where the $[\mathrm{C}$ II] emission peaks. The processing of the Haro 11 observations is described in Cormier et al. (2012).

The data reduction and analysis were performed with SMART v8.2.2 (Lebouteiller et al. 2010; Higdon et al. 2004). IRS high-resolution observations are strongly affected by bad pixels in the detectors. Lacking the observation of an offset field for NGC 625 (and Haro 11), the quality of the spectra mainly relies on the pixel-cleaning step (performed with IRSCLEAN ${ }^{6}$ ) and on the comparison of the two spectra obtained at the two nod positions. The high-resolution spectra were obtained from the full-slit extraction, assuming both point-like and extended source calibrations, since NGC 625 is extended in the MIR bands while the $\mathrm{H}_{2}$ emission seems to be dominated by one knot.

\footnotetext{
6 http://irsa.ipac.caltech.edu/data/SPITZER/docs/ dataanalysistools/tools/irsclean/
} 

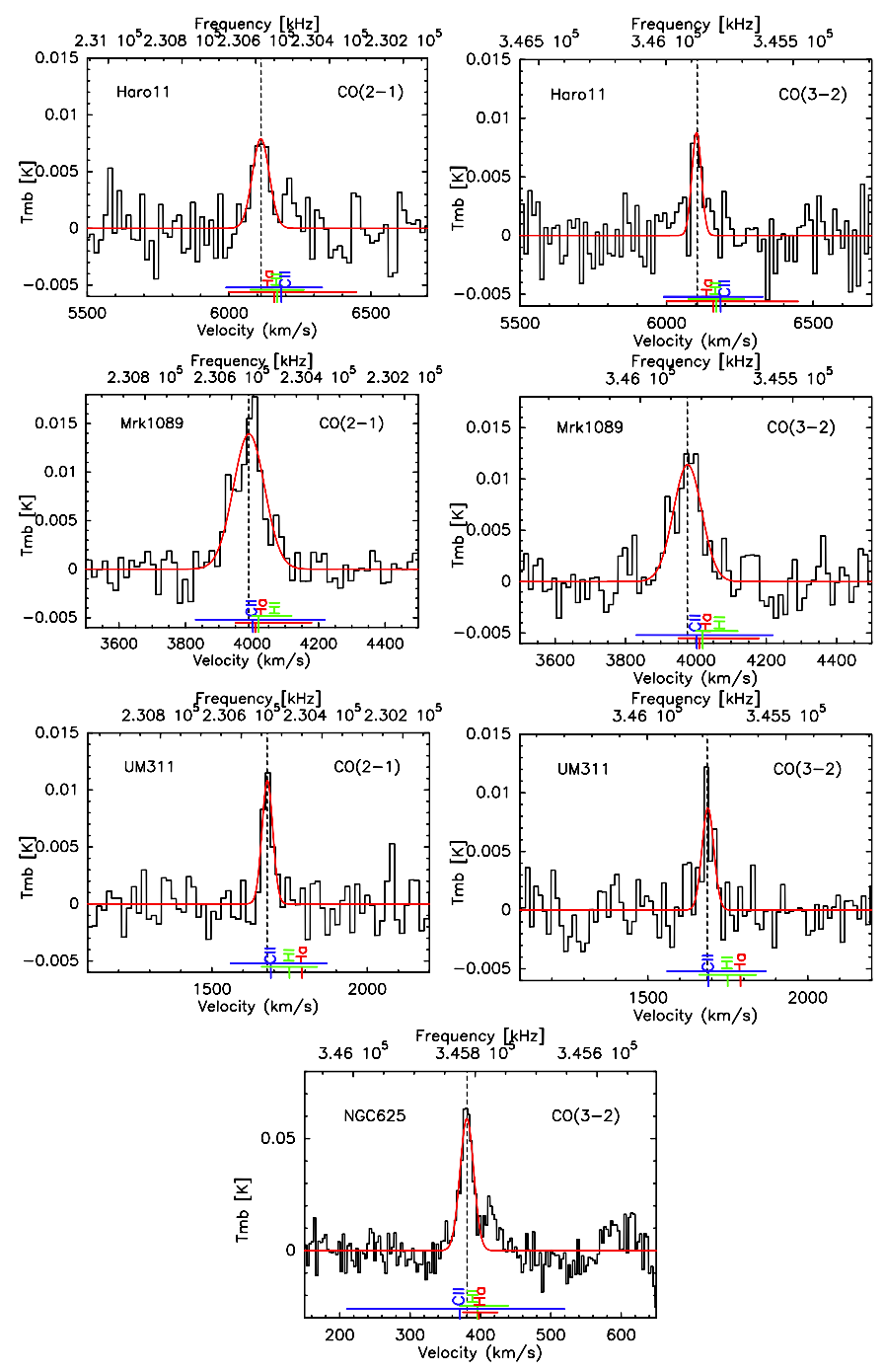

Fig. 3. APEX/SHeFI spectra $\left(T_{\mathrm{mb}}\right)$ of the $\mathrm{CO}(2-1)($ left $)$ and $\mathrm{CO}(3-2)$ (right) lines at $230 \mathrm{GHz}$ and $345 \mathrm{GHz}$, in Haro 11, Mrk 1089, UM 311, and NGC 625 (from top to bottom). The spectra are rebinned to a common resolution of $\sim 10-15 \mathrm{~km} \mathrm{~s}^{-1}$ (and $3 \mathrm{~km} \mathrm{~s}^{-1}$ for NGC 625). Central velocities of [C II], $\mathrm{HI}$, and $\mathrm{H} \alpha$, and their dispersions are also indicated in blue, green, and red, respectively.

We applied a first-order polynomial function fit to the baseline and a Gaussian profile to fit the line. We also added $10 \%$ of the line flux to the total uncertainties in order to take calibration uncertainties into account. The SH spectrum was multiplied by a factor 1.4 and 2 for the point-like and extended calibrations, respectively, to match the continuum level of the LH spectrum (larger aperture). Final fluxes were taken as the average of the fluxes obtained with the two nods and two different calibrations. The IRS observation of NGC 625 points toward the main starburst region where the $[\mathrm{C}$ II] and IR peaks are located, east of the location of the $\mathrm{CO}(2-1)$ and $\mathrm{CO}(3-2)$ pointings (see the IRS footprint on Fig. 1). The IRS slit captures most, but probably not all, of the $\mathrm{H}_{2}$ emission since NGC 625 is extended in the Spitzer and Herschel broad bands. To estimate the total flux (over the entire galaxy), we scaled the $\mathrm{H}_{2}$ emission to that of the total $24 \mu \mathrm{m}$ Spitzer/MIPS emission (Bendo et al. 2012), multiplying all line fluxes by another factor 1.5. Individual spectra of the $\mathrm{H}_{2}$ lines are displayed in Fig. 6, and the line fluxes are given in Table 4. For Haro 11, we used the fluxes from Cormier et al. (2012) but re-estimated a more robust upper limit on the $S(0)$
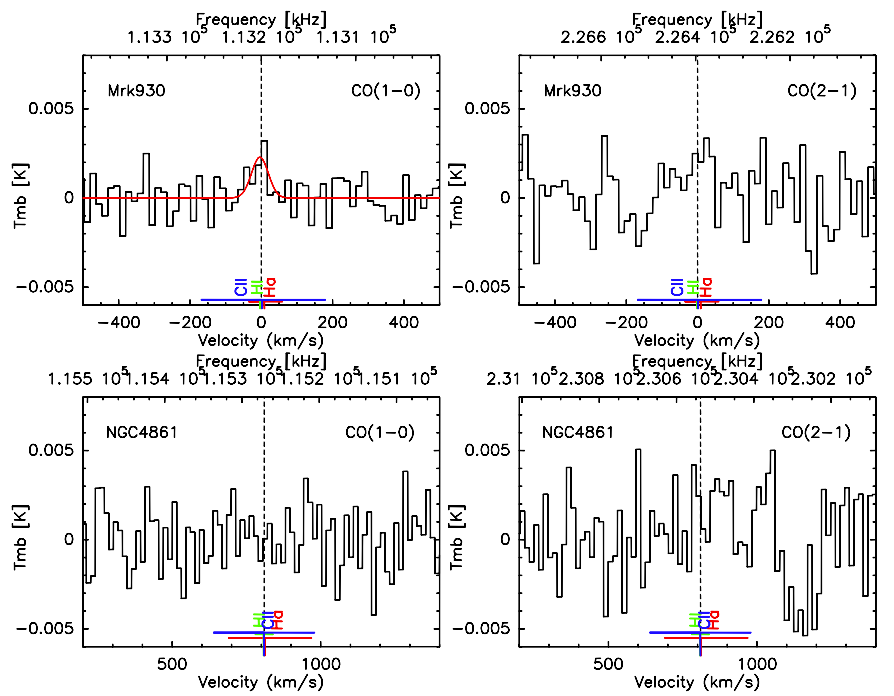

Fig. 4. IRAM- $30 \mathrm{~m}$ spectra $\left(T_{\mathrm{mb}}\right)$ of the $\mathrm{CO}(1-0)$ (left $)$ and $\mathrm{CO}(2-1)$ (right) lines at $115 \mathrm{GHz}$ and $230 \mathrm{GHz}$, in Mrk930 (top) and NGC 4861 (bottom). The spectra are rebinned to a common resolution of $\sim 15 \mathrm{~km} \mathrm{~s}^{-1}$. Central velocities of [C II], HI, and $\mathrm{H} \alpha$, and their dispersions are also indicated in blue, green, and red, respectively.

line by considering $3 \times \mathrm{rms} \times F W H M_{\text {inst }} / \operatorname{sqrt}($ nres $)$, where $n r e s$ is the number of resolution elements in the instrumental FWHM.

The $\mathrm{H}_{2} \mathrm{~S}(1) 17.03 \mu \mathrm{m}$ and $\mathrm{S}(2) 12.28 \mu \mathrm{m}$ lines are detected in NGC 625 (Table 4), while we measure only an upper limit for the $\mathrm{S}(0) 28.22 \mu \mathrm{m}$ line, and the $\mathrm{S}(3) 9.66 \mu \mathrm{m}$ line falls outside of the observed wavelength range. The total luminosity of the two detected $\mathrm{H}_{2}$ lines is $L_{\mathrm{H}_{2}}=1.2 \times 10^{4} L_{\odot}$ for NGC 625 . In the IRS spectrum of Haro 11, the S(1), S(2), and S(3) lines are detected, while the $S(0)$ line is undetected. The total luminosity of the three detected lines is $L_{\mathrm{H}_{2}}=8.6 \times 10^{6} L_{\odot}$.

\section{Analysis of the $\mathrm{CO}$ observations}

\subsection{Line profiles and velocities}

We show the observed CO line profiles in Figs. 2 to 4. For comparison, we indicate below each $\mathrm{CO}$ spectrum the line centers of other relevant tracers: $\mathrm{H} \alpha, \mathrm{HI}$, and [C II], as well as the velocity dispersion, when known. This dispersion measure includes broadening due to line fitting uncertainties (typically a few $\left.\mathrm{km} \mathrm{s}^{-1}\right)$ and the instrumental line width $\left(240 \mathrm{~km} \mathrm{~s}^{-1}\right.$ for [C II] with Herschel/PACS), which could blend several emission components. In those cases where mapped observations are available from the literature, we also include the range of observed peak positions across the source in the dispersion measure. Typical errors on the $\mathrm{CO}$ line centers are of $5-10 \mathrm{~km} \mathrm{~s}^{-1}$. H $\alpha$ velocity information is taken from the following references: James et al. (2013) for Haro 11, Rubin et al. (1990) for Mrk 1089, Pérez-Montero et al. (2011) for Mrk 930, Van Eymeren et al. (2009) for NGC 4861, Marlowe et al. (1997) for NGC 625, and Terlevich et al. (1991) for UM311. H I velocity information is taken from MacHattie et al. (2014) for Haro 11, Williams et al. (1991) for Mrk 1089, Thuan et al. (1999) for Mrk 930, Van Eymeren et al. (2009) for NGC 4861, Cannon et al. (2004) for NGC 625, and Smoker et al. (2000) for UM 311.

For Haro 11, we observe shifts in the velocity centers of the different tracers that could be due to the fact that the peak emission regions for atoms/ions and molecules are not cospatial. Sandberg et al. (2013) find a blueshift of $\sim 44 \mathrm{~km} \mathrm{~s}^{-1}$ of 

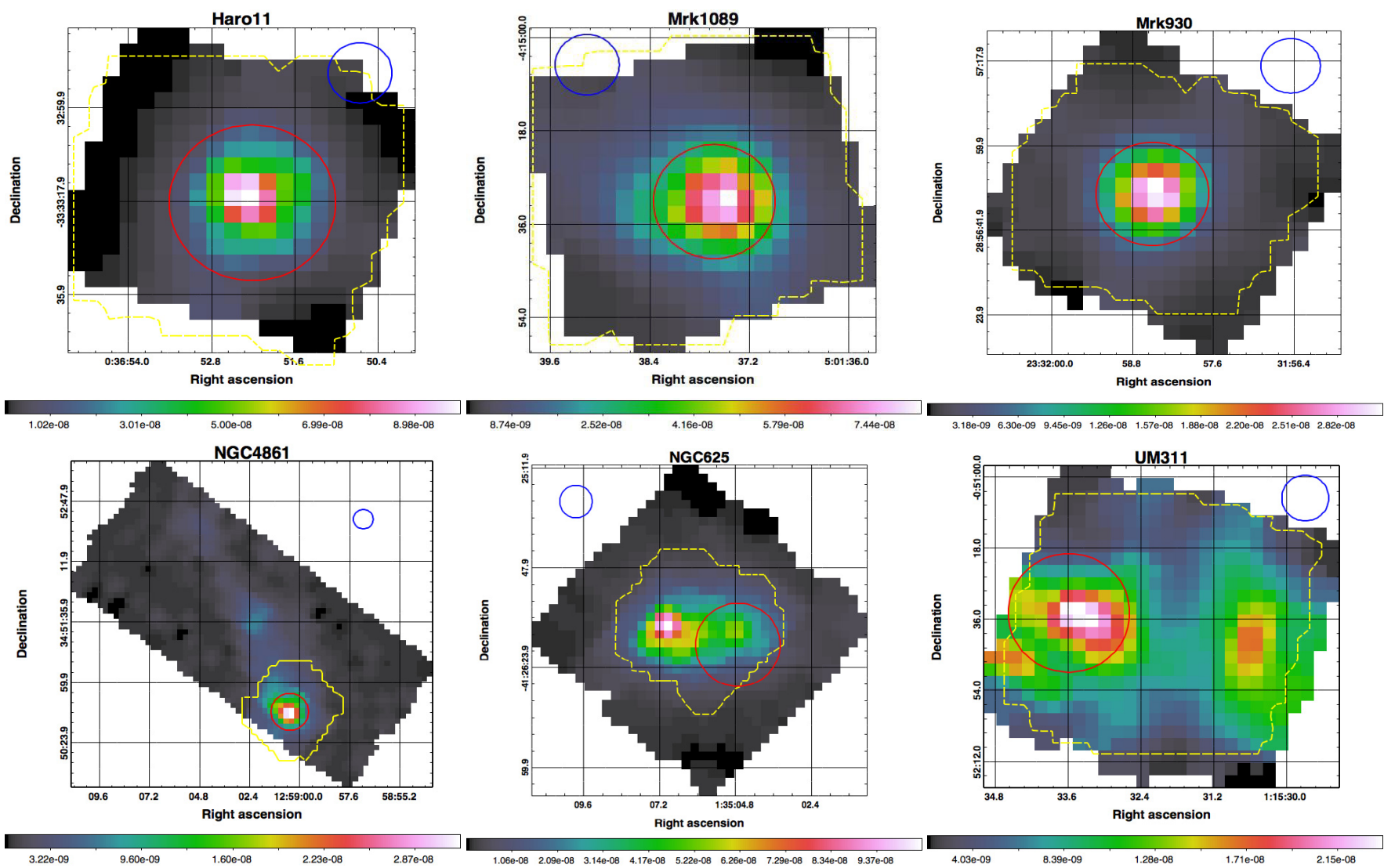

Fig. 5. Herschel/PACS [C II] $157 \mu \mathrm{m}$ intensity maps (units of $\left.\mathrm{W} \mathrm{m}^{-2} \mathrm{sr}^{-1}\right)$. The PACS beam is indicated in blue $\left(\sim 11.5^{\prime \prime}\right)$ and the CO $(1-0)$ aperture used to integrate the flux in red. For NGC 625, the aperture is not centered on the [C II] peak but on the same region covered by the $\mathrm{CO}(2-1)$ and $\mathrm{CO}(3-2)$ observations. The yellow contours show the area mapped in the [O I] $63 \mu \mathrm{m}$ line by PACS.

Table 3. Herschel/PACS [C II] $157 \mu \mathrm{m}$ line observations and fluxes.

\begin{tabular}{|c|c|c|c|c|c|c|c|c|c|}
\hline Galaxy & Program & Line & $\mathrm{OD}^{(a)}$ & OBSID & Map area & $V$ & $\Delta V^{(b)}$ & Total flux & Flux in $\mathrm{CO}(1-0)$ area ${ }^{(c)}$ \\
\hline \multirow[t]{2}{*}{ Haro 11} & DGS & [C II $]$ & 409 & 1342199236 & $51^{\prime \prime} \times 51^{\prime \prime}$ & 6193 & 160 & $6.48 \pm 0.02$ & $5.28 \pm 0.01$ \\
\hline & & {$[\mathrm{O} I]$} & 409 & 1342199237 & $51^{\prime \prime} \times 51^{\prime \prime}$ & 6184 & 140 & $6.45 \pm 0.10$ & $5.34 \pm 0.02$ \\
\hline \multirow[t]{2}{*}{ Mrk 1089} & DGS & [C II] & 690 & 1342217859 & $51^{\prime \prime} \times 51^{\prime \prime}$ & 4020 & - & $8.84 \pm 0.14$ & $4.08 \pm 0.01$ \\
\hline & & {$[\mathrm{OI}]$} & 690 & 1342217861 & $53^{\prime \prime} \times 53^{\prime \prime}$ & 4022 & 80 & $2.83 \pm 0.06$ & $1.48 \pm 0.01$ \\
\hline \multirow[t]{2}{*}{ Mrk 930} & DGS & {$[\mathrm{C}$ II $]$} & 607 & 1342212520 & $51^{\prime \prime} \times 51^{\prime \prime}$ & 5486 & 100 & $2.31 \pm 0.55$ & $1.40 \pm 0.01$ \\
\hline & & [OI] & 607 & 1342212518 & $47^{\prime \prime} \times 47^{\prime \prime}$ & 5488 & 120 & $1.58 \pm 0.10$ & $0.89 \pm 0.01$ \\
\hline \multirow[t]{2}{*}{ NGC 4861} & SHINING & [C II] & 549 & 1342208902 & $1.4^{\prime} \times 3.6^{\prime}$ & 801 & - & $6.96 \pm 0.26$ & $1.39 \pm 0.01$ \\
\hline & & [O I] & 745 & 1342221887 & $47^{\prime \prime} \times 47^{\prime \prime}$ & 799 & 40 & $1.95 \pm 0.07$ & $1.23 \pm 0.01$ \\
\hline \multirow[t]{2}{*}{ NGC 625} & SHINING & [C II $]$ & 754 & 1342222218 & $1.4^{\prime} \times 1.4^{\prime}$ & 383 & - & $16.9 \pm 0.15$ & $4.07 \pm 0.01$ \\
\hline & & {$[\mathrm{O} \mathrm{I}]$} & 754 & 1342222217 & $47^{\prime \prime} \times 47^{\prime \prime}$ & 388 & 100 & $4.85 \pm 0.07$ & $1.25 \pm 0.10$ \\
\hline \multirow[t]{2}{*}{ UM 311} & DGS & [C II $]$ & 621 & 1342213288 & $1.2^{\prime} \times 1.2^{\prime}$ & 1698 & - & $9.98 \pm 0.17$ & $2.23 \pm 0.05$ \\
\hline & & {$[\mathrm{OI}]$} & 621 & 1342213291 & $1.0^{\prime} \times 1.0^{\prime}$ & 1683 & 75 & $3.28 \pm 0.14$ & $1.28 \pm 0.28$ \\
\hline
\end{tabular}

Notes. Fluxes are given in $10^{-16} \mathrm{~W} \mathrm{~m}^{-2}$ and velocities in $\mathrm{km} \mathrm{s}^{-1}$ in the LSR reference frame. The uncertainties on the flux quoted here are those associated with the line fitting and do not take into account the $30 \%$ calibration errors. ${ }^{(a)}$ Observation Day of the Herschel mission. ${ }^{(b)}$ Intrinsic broadening of the line in $\mathrm{km} \mathrm{s}^{-1}$. ${ }^{(c)}$ The aperture over which the fluxes are integrated are shown in Fig. 5.

the neutral gas relative to the ionized gas toward knot $\mathrm{B}$ and a redshift of $\sim 32 \mathrm{~km} \mathrm{~s}^{-1}$ toward knot $\mathrm{C}$. This indicates that the bulk of the cold gas emission could be associated with knot B. Interestingly, the PACS lines display rotation along the northsouth axis and have broad profiles. The full width at half maximum (FWHM) of the FIR lines are larger than the PACS instrumental line widths. Subtracting the instrumental line width $\left(240 \mathrm{~km} \mathrm{~s}^{-1}\right.$ for [C II] and $90 \mathrm{~km} \mathrm{~s}^{-1}$ for [O I] $\left.63 \mu \mathrm{m}\right)$ quadratically, the intrinsic line width of $[\mathrm{C} \mathrm{II}]$ is $\sim 160 \mathrm{~km} \mathrm{~s}^{-1}$, while those of [O III] and [O I] are 250 and $140 \mathrm{~km} \mathrm{~s}^{-1}$, respectively, which is much larger than the CO line width of $\sim 60 \mathrm{~km} \mathrm{~s}^{-1}$. The [O III] broadening agrees with that of $\mathrm{H} \alpha$, found as large as $\sim 280 \mathrm{~km} \mathrm{~s}^{-1}$ by James et al. (2013). This confirms that the emission of the FIR lines arises from a larger region than the $\mathrm{CO}$ emission. The FIR lines are also too broad to be solely due to rotation and probably trace the presence of outflows.

For UM 311, important velocity shifts are observed for the different tracers, which are probably due to the crowding of 

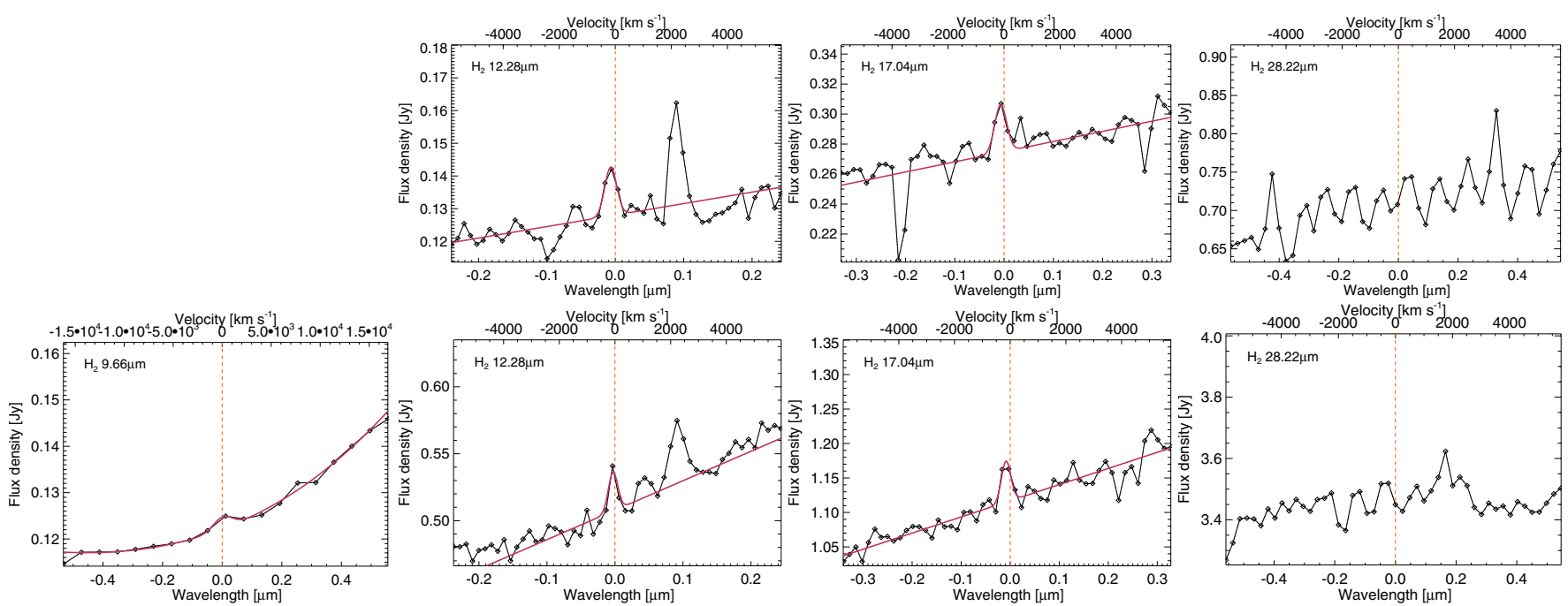

Fig. 6. Spitzer/IRS spectra of the $\mathrm{H}_{2}$ lines at 9.66, 12.28, 17.04, and $28.22 \mu \mathrm{m}$ in NGC 625 (top row) and Haro 11 (bottom row). The red curve indicates the fit to the line when detected.

Table 4. Spitzer/IRS $\mathrm{H}_{2}$ observations and fluxes.

\begin{tabular}{lccc}
\hline \hline $\begin{array}{l}\text { Galaxy } \\
\text { Observing mode } \\
\text { AORKey }\end{array}$ & $\begin{array}{c}\text { NGC 625 } \\
\text { staring } \\
5051904\end{array}$ & $\begin{array}{c}\text { NGC 625 }_{\text {total }^{b}} \\
\text { Line flux: }\end{array}$ & $\begin{array}{c}\text { Haro 11 } \\
\text { staring } \\
9007104\end{array}$ \\
H2 S(0) $28.22 \mu \mathrm{m}$ & $<18.4$ & $<27.6$ & $<24.9$ \\
$\mathrm{H}_{2} \mathrm{~S}(1) 17.03 \mu \mathrm{m}$ & $10.8 \pm 2.6$ & $16.2 \pm 3.8$ & $16.8 \pm 5.5$ \\
$\mathrm{H}_{2} \mathrm{~S}(2) 12.28 \mu \mathrm{m}$ & $6.39 \pm 1.40$ & $9.58 \pm 2.50$ & $10.1 \pm 1.3$ \\
$\mathrm{H} 2 \mathrm{~S}(3) 9.66 \mu \mathrm{m}$ & - & - & $12.1 \pm 1.8$ \\
\hline
\end{tabular}

Notes. Fluxes are in $10^{-18} \mathrm{~W} \mathrm{~m}^{-2}$ and upper limits are given at a $3 \sigma$ level. ${ }^{(a)}$ Estimated total $\mathrm{H}_{2}$ emission from the galaxy by scaling each flux measured in the IRS slit to the $24 \mu \mathrm{m}$ emission of the galaxy.

the region, while for the other galaxies, $\mathrm{CO}$ and the other tracers peak at the same velocity overall. Mrk 1089 has broad CO profiles and the signal-to-noise is high enough in the APEX $\mathrm{CO}(2-1)$ and $\mathrm{CO}(3-2)$ spectra to clearly distinguish two velocity components, which are also present in the data from Leon et al. (1998). The spectral resolution of PACS does not allow us to resolve the separate narrow components seen in the $\mathrm{CO}$ profiles. Our [C II] velocity map displays rotation, with redshifted emission east of the nucleus and blueshifted emission west of the nucleus. This agrees with the velocity analysis of $\mathrm{HI}$ and $\mathrm{H} \alpha$, although the velocity fields are more irregular in the HCG 31 complex as a whole. In NGC 625, the CO(1-0) spectra have a single CO component of width $\sim 70 \mathrm{~km} \mathrm{~s}^{-1}$. This emission may not be cospatial with the one seen in the ancillary $\mathrm{CO}(2-1)$ and $\mathrm{CO}(3-2)$ spectra (see Sect. 2.2), which are narrower (Table 2). While $\mathrm{H} \alpha$ and [C II] peak spatially where the current star formation episode is taking place and where we detect $\mathrm{CO}$ with Mopra, H I peaks $\sim 300$ pc east (Cannon et al. 2004), where the second peak of [C II] is located and where the APEX and JCMT observations point.

To summarize, the $\mathrm{CO}$ line detections are consistent with the velocities as obtained from $\mathrm{H} \alpha, \mathrm{HI}$, and [C II]. Moreover, for a given galaxy, the different $\mathrm{CO}$ transitions exhibit the same line center and width (except $\mathrm{CO}(1-0)$ in NGC 625) within the uncertainties, showing that they come from the same component. This allows us to study CO line ratios (Sect. 4.1.1).

\subsection{Estimating the total CO emission}

One of the complications in analyzing the various $\mathrm{CO}$ observations is the difference in beam sizes used for each transition. A larger beam may encompass more molecular clouds than probed by a smaller beam and thus hamper the comparison of the transitions with each other. A further complication stems from the possibility of CO emission outside the observed area if the galaxy is more extended than any of the beams, which influences the results when comparing $\mathrm{CO}$ properties to global properties (e.g., H I mass). Here we investigate how using different beam sizes affects the derived intensities.

We have a complete coverage of the $100 \mu \mathrm{m}$ dust emission from Herschel for each galaxy at our disposal (Fig. 7; Rémy-Ruyer et al. 2013). If we assume that the distribution of the CO emission follows that of the $100 \mu \mathrm{m}$ dust continuum, we can roughly estimate the fraction of $\mathrm{CO}$ emission measured in each $\mathrm{CO}$ beam relative to the total $\mathrm{CO}$ emission. In principle, one should first subtract the H I column density for such approach. Here, we just assume a flat $\mathrm{H}$ I distribution. The spatial correlation between $\mathrm{CO}$ and the dust may not follow the same distribution on small scales in the Galaxy, but on larger scales in external galaxies, observations show a correlation between $\mathrm{CO}$ and IR emission (e.g., Tacconi \& Young 1987; Sanders et al. 1991). The dust emission in the spectral energy distribution (SED) of our dwarf galaxies typically peaks between $70-100 \mu \mathrm{m}$, which makes the $100 \mu \mathrm{m}$ emission a relatively good proxy for the dust column density. We convolve the $100 \mu \mathrm{m}$ maps with a 2D Gaussian. The position and width of the Gaussian are chosen to correspond to the position and width ${ }^{7}$ of the respective $\mathrm{CO}$ observations. The $\mathrm{CO}$ fractions that we estimate this way are listed in Table 5.

For Haro 11, Mrk 1089, and Mrk 930, we have recovered the bulk of the $\mathrm{CO}$ emission, and the $\mathrm{CO}$ luminosities that we report in Table 2 can be considered as total values. The fractions of the $\mathrm{CO}$ emission captured in the different $\mathrm{CO}$ beams agree within $20 \%$, and comparing the CO intensities from one transition with another should be quite reliable (except for Mrk930 but we only have a limit on its ratio). On the other hand, NGC 4861, NGC 625, and UM 311 are more extended and have complex shapes, and a large fraction of the total $100 \mu \mathrm{m}$ flux is emitted

7 The FWHM used equals $\sqrt{F W H M(\mathrm{CO})^{2}-F W H M(\mathrm{PACS})^{2}}$. 

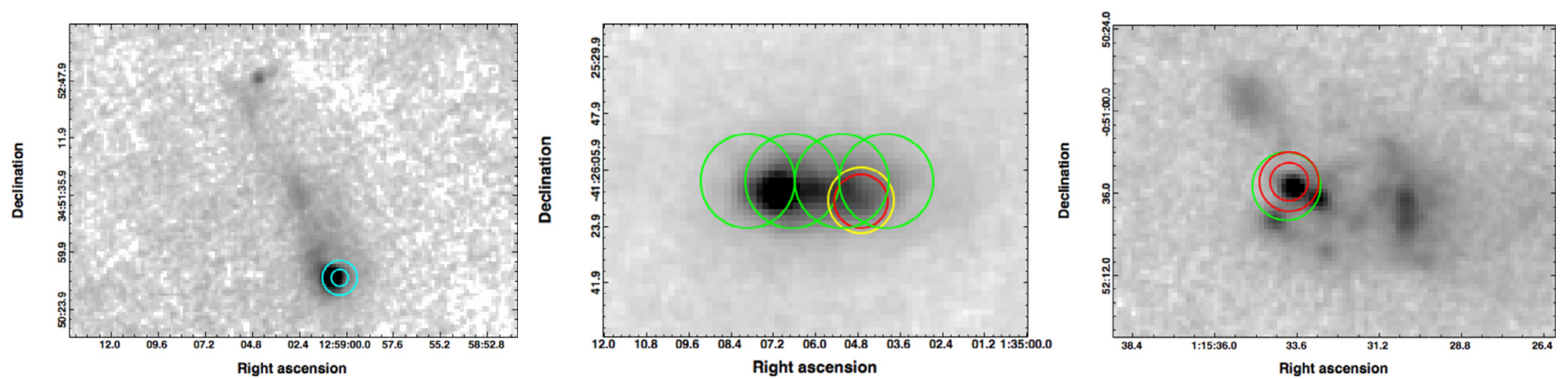

Fig. 7. Herschel PACS $100 \mu \mathrm{m}$ maps of NGC 4861 (left), NGC 625 (middle), and UM 311 (right), with the CO beams (Mopra in green, APEX in red, IRAM in blue, and the JCMT in yellow; see Fig. 1 for details).

Table 5. Estimated CO fractions (in \%).

\begin{tabular}{cccc}
\hline \hline Galaxy & 1-0/total & 2-1/total & 3-2/total \\
\hline Haro 11 & 85 & 81 & 65 \\
Mrk 1089 & 56 & 63 & 44 \\
Mrk 930 & 62 & 28 & - \\
NGC 4861 & 28 & 10 & - \\
NGC 625 & 27 & 17 & 11 \\
UM 311 & 21 & 18 & 10 \\
\hline
\end{tabular}

Notes. Fraction of the $100 \mu \mathrm{m}$ flux from Herschel measured in each $\mathrm{CO}$ beam relative to the total $100 \mu \mathrm{m}$ flux - where the distribution of the $100 \mu \mathrm{m}$ dust emission is used as a proxy for the COemission.

outside the $\mathrm{CO}$ beams. For these galaxies, we apply correction factors to the observed $\mathrm{CO}$ fluxes to estimate the total $\mathrm{CO}$ fluxes (Table 2). For NGC 4861, in which we targeted only the main $\mathrm{H}$ II region, this correction factor is 3.5. For UM 311, the correction factor is 5 because of $100 \mu \mathrm{m}$ contamination from the two neighboring spiral galaxies. In this case, it is unclear whether the $100 \mu \mathrm{m}$ emission can be used to calculate beam correction factors since these systems may or may not be hosting CO clumps. For the sake of consistency, we apply this correction factor and estimate the total $\mathrm{CO}$ emission for the entire system. The $\mathrm{CO}$ line ratios are also uncertain by a factor of $\sim 2$. For NGC 625 , we consider a single Mopra beam at the position of the $\mathrm{CO}(3-2)$ pointing. The difference between the $100 \mu \mathrm{m}$ fluxes integrated within the individual $\mathrm{CO}(1-0)$ and $\mathrm{CO}(3-2)$ beams is a factor of 2 . The reason for this difference in NGC 625 is that the $\mathrm{CO}(3-2)$ observation does not point toward the peak of dust emission, and the $\mathrm{CO}(1-0)$ and $\mathrm{CO}(3-2)$ beam sizes are quite different. For the total $\mathrm{CO}$ emission of NGC 625, we consider the emission in all the $\mathrm{CO}(1-0)$ Mopra pointings, which cover the full extent of the galaxy seen in the dust continuum. When doing so, only $10 \%$ of the $100 \mu \mathrm{m}$ emission is emitted outside our $\mathrm{CO}(1-0)$ coverage.

These rough estimates of possible $\mathrm{CO}$ emission outside of the respective $\mathrm{CO}$ beams show that there are uncertainties of about a factor of 2 when comparing $\mathrm{CO}$ lines with each other, and a factor of $\sim 3$ for NGC 4861 and UM 311 when considering global quantities, such as molecular masses.

\section{Physical conditions in the molecular clouds}

In this section, we investigate the conditions (temperature, density, mass) characterizing the CO-emitting gas in our galaxies. First we discuss line ratios and derive total molecular masses using two values of the CO-to- $\mathrm{H}_{2}$ conversion factor (Galactic and metallicity-scaled) and dust measurements. Then we compare
Table 6. CO line ratios.

\begin{tabular}{cccc}
\hline \hline Galaxy & $3-2 / 1-0$ & $3-2 / 2-1$ & $2-1 / 1-0$ \\
\hline Haro 11 & & & \\
point-like & $>0.23$ & $0.38 \pm 0.23$ & $>0.60$ \\
extended & $>0.71$ & $0.89 \pm 0.53$ & $>0.79$ \\
Mrk 1089 & & & \\
point-like & $0.30 \pm 0.13$ & $0.30 \pm 0.13$ & $1.00 \pm 0.31$ \\
extended & $0.46 \pm 0.19$ & $0.70 \pm 0.31$ & $0.65 \pm 0.20$ \\
Mrk 930 & & & \\
point-like & - & - & $<0.69$ \\
extended & - & - & $<2.51$ \\
NGC 625 & & & $>0.45$ \\
point-like & $>0.29$ & $0.64 \pm 0.30$ & $>1.02$ \\
extended & $>0.90$ & $0.88 \pm 0.42$ & \\
UM 311 & & & $>0.58$ \\
point-like & $>0.21$ & $0.36 \pm 0.21$ & \\
extended & $>0.66$ & $0.85 \pm 0.49$ & $>0.77$ \\
\hline
\end{tabular}

the observations with the predictions of the code RADEX and contrast our $\mathrm{CO}$ results with the analysis of the warm $\mathrm{H}_{2}$ gas.

\subsection{Empirical diagnostics}

\subsubsection{Line ratios}

COline intensity ratios give an insight into the conditions of the gas. Typical ratios are $\mathrm{CO}(2-1) / \mathrm{CO}(1-0) \sim 0.8$ and $\mathrm{CO}(3-2) / \mathrm{CO}(1-0) \sim 0.6$ in nearby spiral galaxies (Leroy et al. 2008; Wilson et al. 2009; Warren et al. 2010), and $\mathrm{CO}(3-2) / \mathrm{CO}(2-1) \sim 0.8$ in Galactic giant molecular clouds (Wilson et al. 1999). In blue compact dwarf galaxies, Sage et al. (1992) find an average ratio of $\mathrm{CO}(2-1) / \mathrm{CO}(1-0) \sim 0.8$, and Meier et al. (2001) derive an average $\mathrm{CO}(3-2) / \mathrm{CO}(1-0)$ ratio of 0.6 in starburst dwarf galaxies. Ratios of $\mathrm{CO}(2-1) / \mathrm{CO}(1-0) \geq 1$ are found in several starbursting compact dwarfs (e.g., Israel 2005) and bright regions of the Magellanic Clouds (Bolatto et al. 2000, 2003), and are usually attributed to the emission arising from smaller, warmer clumps, where $\mathrm{CO}(1-0)$ may not trace the total molecular gas.

The line ratios are calculated from the observed $\mathrm{CO}$ line intensities $I_{\mathrm{CO}}\left(\mathrm{K} \mathrm{km} \mathrm{s}^{-1}\right)$, and solid angles of the source $\left(\Omega_{\text {source }}\right)$ and of the beam $\left(\Omega_{\text {beam }}\right)$, following Meier et al. (2001):

$\mathrm{CO} 2-1 / 1-0=\frac{I_{\mathrm{CO} 2-1}}{I_{\mathrm{CO} 1-0}} \times \frac{\Omega_{\text {source }}+\Omega_{\text {beam, CO 2-1 }}}{\Omega_{\text {source }}+\Omega_{\text {beam, CO 1-0 }}}$.

The source sizes are unknown since we do not resolve individual molecular clouds. The assumed spatial distribution of the emission influences the derived CO line ratios (see also Sect. 3.2). 
Table 7. $\mathrm{H}_{2}$ masses using $\mathrm{CO}$ observations, dust measurements, and models.

\begin{tabular}{|c|c|c|c|c|c|c|c|c|c|}
\hline \multirow[t]{3}{*}{ Galaxy } & \multicolumn{5}{|c|}{ From CO observations } & \multirow{3}{*}{$\frac{\text { From dust }}{M_{\mathrm{H}_{2}}\left(M_{\odot}\right)}$} & \multirow{3}{*}{$\frac{\text { From LTE }}{M_{\mathrm{H}_{2}}^{\text {warm }}\left(M_{\odot}\right)}$} & \multirow{2}{*}{\multicolumn{2}{|c|}{$\begin{array}{c}\text { From Cloudy } \\
M_{\mathrm{H}_{2}}\left(M_{\odot}\right) \\
\end{array}$}} \\
\hline & \multirow[t]{2}{*}{$X_{\mathrm{CO}} / X_{\mathrm{CO}, \mathrm{gal}}^{(a)}$} & \multicolumn{4}{|c|}{$M_{\mathrm{H}_{2}}\left(M_{\odot}\right)$} & & & & \\
\hline & & $\mathrm{CO}(1-0)$ & $R_{21}=0.8^{(b)}$ & $R_{31}=0.6^{(c)}$ & Average & & & CO-free & CO-traced \\
\hline \multirow[t]{2}{*}{ Haro 11} & 1 & $<4.4 \times 10^{8}$ & $3.3 \times 10^{8}$ & $1.7 \times 10^{8}$ & $2.5 \times 10^{8}$ & $3.6 \times 10^{9}$ & $>3.6 \times 10^{6}<2.4 \times 10^{8}$ & $1.2 \times 10^{8}$ & $1.6 \times 10^{9}$ \\
\hline & 10 & $<4.4 \times 10^{9}$ & $3.3 \times 10^{9}$ & $1.7 \times 10^{9}$ & $2.5 \times 10^{9}$ & - & & & \\
\hline \multirow[t]{2}{*}{ Mrk 1089} & 1 & $3.1 \times 10^{8}$ & $3.9 \times 10^{8}$ & $1.6 \times 10^{8}$ & $2.9 \times 10^{8}$ & $8.3 \times 10^{8}$ & & & \\
\hline & 16 & $4.9 \times 10^{9}$ & $6.2 \times 10^{9}$ & $2.5 \times 10^{9}$ & $4.5 \times 10^{9}$ & - & & & \\
\hline \multirow[t]{2}{*}{ Mrk 930} & 1 & $3.1 \times 10^{7}$ & $<9.8 \times 10^{7}$ & - & $3.1 \times 10^{7}$ & $1.0 \times 10^{9}$ & & & \\
\hline & 22 & $6.8 \times 10^{8}$ & $<2.1 \times 10^{9}$ & - & $6.8 \times 10^{8}$ & - & & & \\
\hline \multirow[t]{2}{*}{ NGC 4861} & 1 & $<7.3 \times 10^{5}$ & $<1.2 \times 10^{6}$ & - & $<7.3 \times 10^{5}$ & - & & & \\
\hline & 42 & $<3.1 \times 10^{7}$ & $<5.1 \times 10^{7}$ & - & $<3.1 \times 10^{7}$ & - & & & \\
\hline Total & 1 & $<2.6 \times 10^{6}$ & - & - & $<2.6 \times 10^{6}$ & $1.7 \times 10^{8}$ & & & \\
\hline \multirow{2}{*}{ NGC 625} & 1 & $<1.9 \times 10^{6}$ & $1.1 \times 10^{6}$ & $9.0 \times 10^{5}$ & $1.0 \times 10^{6}$ & - & & & \\
\hline & 9 & $<1.7 \times 10^{7}$ & $9.7 \times 10^{6}$ & $8.2 \times 10^{6}$ & $9.0 \times 10^{6}$ & - & & & \\
\hline Total & 1 & $5.0 \times 10^{6}$ & - & - & $5.0 \times 10^{6}$ & $9.4 \times 10^{7}$ & $>5.8 \times 10^{3}<5.7 \times 10^{5}$ & & \\
\hline \multirow[t]{2}{*}{ UM 311} & 1 & $<2.7 \times 10^{7}$ & $2.0 \times 10^{7}$ & $9.4 \times 10^{6}$ & $1.5 \times 10^{7}$ & - & & & \\
\hline & 4 & $<1.2 \times 10^{8}$ & $8.5 \times 10^{7}$ & $4.1 \times 10^{7}$ & $6.3 \times 10^{7}$ & - & & & \\
\hline Total & 1 & $<1.3 \times 10^{8}$ & $9.8 \times 10^{7}$ & - & $9.8 \times 10^{7}$ & $3.3 \times 10^{9}$ & & & \\
\hline
\end{tabular}

Notes. The $\mathrm{H}_{2}$ masses from $\mathrm{CO}$ observations are measured within the $\mathrm{CO}$ beams. For extended galaxies, we also consider the estimated total CO emission (see Sect. 3.2 for details). ${ }^{(a)}$ Ratio of our adopted $X_{\mathrm{CO}}$ value to the Galactic value $X_{\mathrm{CO}, \text { gal }}$. To bracket the range of molecular gas masses, there are two lines for each galaxy corresponding to the molecular masses derived with two values of the $X_{\mathrm{CO}}$ factor: $X_{\mathrm{CO}}=X_{\mathrm{CO}, \mathrm{gal}}($ lower mass case) and $X_{\mathrm{CO}}=X_{\mathrm{CO}, Z} \propto Z^{-2}$ (upper mass case). ${ }^{(b)} R_{21}$ is the $\mathrm{CO}(2-1) / \mathrm{CO}(1-0)$ ratio. ${ }^{(c)} R_{31}$ is the $\mathrm{CO}(3-2) / \mathrm{CO}(1-0)$ ratio.

Therefore we derive line ratios in two cases: (1) if the source is point-like $\left(\Omega_{\text {source }} \ll \Omega_{\text {beam }}\right)$ or (2) if the source is much larger than the beam $\left(\Omega_{\text {beam }} \ll \Omega_{\text {source }}\right)$. The latter case also applies if the source is not resolved but the CO emission comes from small clumps scattered across the beam and fills the beam (no beam dilution). Values of the line ratios are summarized in Table 6.

For the sample, we find an average $\mathrm{CO}(3-2) / \mathrm{CO}(2-1)$ ratio of $\sim 0.6$ (measured on the detections only). Ratios of $\mathrm{CO}(3-2) / \mathrm{CO}(1-0)$ and $\mathrm{CO}(2-1) / \mathrm{CO}(1-0)$ are respectively $\geq 0.2$ and $\geq 0.6$ across the sample. Although there can be significant uncertainties due to the different beam sizes (see Sect. 3.2) and unknown source distribution, overall, we find no evidence for significantly different $\mathrm{CO}$ line ratios in our sample compared to normal-metallicity environments. Unlike resolved lowmetallicity environments where COline ratios can be high due to locally warmer gas temperatures, the line ratios (hence CO temperature) in our compact dwarf galaxies are moderate.

\subsubsection{Molecular gas mass from $\mathrm{CO}$ observations}

The molecular gas mass is usually derived from $\mathrm{CO}$ observations as a tracer of the cold $\mathrm{H}_{2}$ using the CO-to- $\mathrm{H}_{2}$ conversion factor $X_{\mathrm{CO}}{ }^{8}$, which relates the observed $\mathrm{CO}$ intensity to the column density of $\mathrm{H}_{2}$ :

$X_{\mathrm{CO}}=N\left(\mathrm{H}_{2}\right) / I_{\mathrm{CO}}\left[\mathrm{cm}^{-2}\left(\mathrm{~K} \mathrm{~km} \mathrm{~s}^{-1}\right)^{-1}\right]$.

The extent to which the conversion from $\mathrm{CO}$ emission to cold $\mathrm{H}_{2}$ reservoir is affected by metallicity depends crucially on the relative proportion of $\mathrm{H}_{2}$ residing at moderate $A_{V}$ compared to the amount of enshrouded molecular gas. Even though both simulations and observations support a change in the CO-to- $\mathrm{H}_{2}$ conversion factor at low metallicities, the exact dependence of $X_{\mathrm{CO}}$

\footnotetext{
8 Dame et al. (2001) report an average value in the Galaxy of $X_{\mathrm{CO} \text {,gal }}=1.8 \times 10^{20} \mathrm{~cm}^{-2}\left(\mathrm{~K} \mathrm{~km} \mathrm{~s}^{-1}\right)^{-1}$, and Fermi measurements give $X_{\mathrm{CO}, \text { gal }} \simeq 2 \times 10^{20} \mathrm{~cm}^{-2}\left(\mathrm{~K} \mathrm{~km} \mathrm{~s}^{-1}\right)^{-1}$ for large-scale complexes (e.g., Ackermann et al. 2011). In the following, we adopt $X_{\mathrm{CO}, \text { gal }}=$ $2 \times 10^{20} \mathrm{~cm}^{-2}\left(\mathrm{~K} \mathrm{~km} \mathrm{~s}^{-1}\right)^{-1}$. These variations in $X_{\mathrm{CO}, \text { gal }}$ are insignificant here compared to the orders of magnitude variations that may prevail in our low-metallicity sample.
}

on metallicity is not empirically well-established. Many physical characteristics other than metallicity, such as the topology of the molecular clouds and the distribution of massive ionizing stars, are also at play. We refer the reader to Bolatto et al. (2013) for a review on the topic. Therefore we consider two cases: one where $X_{\mathrm{CO}}$ is equal to the Galactic value $X_{\mathrm{CO}, \mathrm{gal}}$, and one where $X_{\mathrm{CO}}$ scales with metallicity (noted $X_{\mathrm{CO}, Z}$ ) following Schruba et al. (2012), $X_{\mathrm{CO}, Z} \propto Z^{-2}$. Although the $X_{\mathrm{CO}}$ conversion factor is calibrated for the $\mathrm{CO}(1-0)$ transition, we also estimate the molecular mass from the $\mathrm{CO}(2-1)$ and $\mathrm{CO}(3-2)$ observations, using the values of 0.8 and 0.6 for the $\mathrm{CO}(2-1) / \mathrm{CO}(1-0)$ and $\mathrm{CO}(3-2) / \mathrm{CO}(1-0)$ ratios respectively (Sect. 4.1.1). For each $\mathrm{CO}$ transition, the source size is taken as its corresponding beam size. For galaxies observed with IRAM (Mrk930 and NGC 4861), we assume the source size to be that of the $\mathrm{CO}(1-0)$ beam since only the $\mathrm{CO}(1-0)$ observations in Mrk 930 leads to a detection. The resulting molecular gas masses of the six galaxies are reported in Table 7.

For Haro 11, our CO detections yield a molecular gas mass of $M\left(\mathrm{H}_{2}\right) \simeq 2.5 \times 10^{8} M_{\odot}\left(\right.$ with $\left.X_{\mathrm{CO}, \mathrm{gal}}\right)$. This value is higher than the previous upper limit of Bergvall et al. (2000), where they estimated $M\left(\mathrm{H}_{2}\right) \leq 10^{8} M_{\odot}$. For Mrk 1089, Leon et al. (1998) found $M\left(\mathrm{H}_{2}\right)=4.4 \times 10^{8} M_{\odot}$, This value is 1.5 times higher than what we derive with $X_{\mathrm{CO} \text {,gal }}$ due to a beam (hence assumed source size) difference, and is below the value that we derive with $X_{\mathrm{CO}, Z}$. There are no other molecular mass estimates for Mrk 930, NGC 625, NGC 4861, and UM 311 in the literature to which we can compare our estimates. The molecular gas masses that we find with $X_{\mathrm{CO} \text {,gal }}$ (denoted $M\left(\mathrm{H}_{2, \mathrm{gal}}\right)$ ) are particularly low compared to, e.g., the IR luminosity or H I reservoir of our galaxies. We find an average ratio (with minimum and maximum values) of $M\left(\mathrm{H}_{2, \mathrm{gal}}\right) / M(\mathrm{HI}) \sim 122_{1.0}^{50} \%$ and $M\left(\mathrm{H}_{2, \mathrm{gal}}\right) / L_{\mathrm{TIR}} \sim 1.0 .0 \%$ (typically $10 \%$ in isolated galaxies; Solomon \& Sage 1988). This stresses how deficient our galaxies are in CO.

\subsubsection{Molecular gas mass from dust measurements}

Other methods based on dust measurements are also used to quantify the total $\mathrm{H}_{2}$ gas, assuming that the gas mass is proportional to the dust mass via a fixed dust-to-gas mass ratio 
Table 8. Dust-derived $X_{\mathrm{CO}}$ factors.

\begin{tabular}{lcccc}
\hline \hline Galaxy & $M_{\text {dust }}\left[M_{\odot}\right]$ & $D / G$ & $X_{\mathrm{CO}, \text { dust }} / X_{\mathrm{CO}, \text { gal }}$ & $X_{\mathrm{CO}, \text { dust }} / X_{\mathrm{CO}, Z}$ \\
\hline Haro 11 & $9.9 \times 10^{6}$ & $10^{-2.7}$ & 14 & 1.4 \\
Mrk 1089 & $2.6 \times 10^{7}$ & $10^{-2.8}$ & 2.8 & 0.2 \\
Mrk 930 & $6.0 \times 10^{6}$ & $10^{-2.8}$ & 33 & 1.5 \\
NGC 4861 & $6.0 \times 10^{5}$ & $10^{-3.0}$ & 65 & 1.6 \\
NGC 625 & $4.5 \times 10^{5}$ & $10^{-2.7}$ & 36 & 3.9 \\
UM 311 & $2.0 \times 10^{7}$ & $10^{-2.5}$ & 44 & 10 \\
\hline
\end{tabular}

Notes. Dust masses are from Rémy-Ruyer et al. (in prep.).

(e.g., Leroy et al. 2011; Sandstrom et al. 2013):

$$
M\left(\mathrm{H}_{2}\right)=M_{\text {dust }} \times[D / G]^{-1}-[M(\mathrm{HI})+M(\mathrm{HII})] \text {. }
$$

We consider quantities within apertures defined from the dust emission and use aperture-corrected $\mathrm{HI}$ masses from Rémy-Ruyer et al. 2014 (Table 9). Except for Haro 11 (H II mass from Cormier et al. 2012), we ignore $M$ (H II) from the equation since there is no ionized gas mass reported in the literature for our galaxies. We note that $M(\mathrm{H}$ II) might be negligible in normal galaxies but might contribute more to the mass budget of dwarf galaxies. For the dust-to-gas mass ratio $(D / G)$, we choose a D/G that scales linearly with metallicity (e.g., Edmunds 2001) and adopt the value $D / G=1 / 150$ for $\mathrm{O} / \mathrm{H}=8.7$ (Zubko et al. 2004). The dust masses are measured in Rémy-Ruyer et al. (in prep.) from a full dust SED modeling (Galliano et al. 2011) up to $500 \mu \mathrm{m}$ with Galactic dust opacities. Rémy-Ruyer et al. (in prep.) show that the thus derived dust masses are not significantly affected by the presence of a possible submm excess. Uncertainties on the dust masses due to the submm excess or a change of grain properties in low-metallicity environments are a factor 2-3 (Galametz et al. 2011; Galliano et al. 2011). We provide the $\mathrm{D} / \mathrm{G}$ and dust masses in Table 8 and the derived $\mathrm{H}_{2}$ masses in Table 7. We find that the $\mathrm{H}_{2}$ masses resulting from this method are systematically higher by one or two orders of magnitude (except in Mrk 1089) than those determined from CO and $X_{\mathrm{CO}, \mathrm{gal}}$.

If we assume that the $\mathrm{H}_{2}$ mass derived from the dust method is the true molecular mass, we can use the observed $\mathrm{CO}$ intensities and calculate the corresponding $X_{\mathrm{CO}}$ value, $X_{\mathrm{CO} \text {,dust }}$. Since the full extent of the $\mathrm{CO}$ emission is unknown for the extended galaxies, we consider the fraction of the $\mathrm{H}_{2}$ mass that would fall in the $\mathrm{CO}(1-0)$ beam (Sect. 3.2). We re-estimate the $\mathrm{CO}(1-0)$ intensities based on the average $\mathrm{H}_{2}$ masses of the three $\mathrm{CO}$ transitions (Table 7) to derive $X_{\mathrm{CO} \text {,dust }}$ (Table 8). The $X_{\mathrm{CO} \text {,dust values are }}$ 10-50 times higher than $X_{\mathrm{CO} \text {,gal }}$, and sometimes even higher than $X_{\mathrm{CO}, Z}$. They scale with metallicity with power-law index $\simeq-2.7$ : $X_{\mathrm{CO} \text {,dust }} \propto(\mathrm{O} / \mathrm{H})^{-2.7}$. This relation with metallicity is steeper than what is found in Leroy et al. (2011) for Local Group galaxies (index of -1.7), and similar to the one found in Israel (2000) (index of -2.5) and in Schruba et al. (2012) for their complete sample of dwarf galaxies (index of -2.8).

The numbers from this method are only given as an indication since they bear large uncertainties. We have done the same exercise on seven additional compact galaxies of the DGS (names in Table 9). These galaxies have $7.75<\mathrm{O} / \mathrm{H}$ and are selected with total detected $\mathrm{CO}$ emission (within $50 \%$, comparing the $[\mathrm{C} \mathrm{II}]$ emission inside and outside the $\mathrm{CO}$ aperture, Cormier et al., in prep.). However, the total gas masses predicted from the dust are often lower than the H I masses (and not only for the lowest metallicity objects). This shows the limitation of this method for compact low-metallicity galaxies that
Table 9. Parameters for the star formation law.

\begin{tabular}{lccccr}
\hline \hline Galaxy & $\begin{array}{c}\text { Metal. } \\
{[\mathrm{O} / \mathrm{H}]} \\
(2)\end{array}$ & $\begin{array}{c}M(\mathrm{HI}) \\
{\left[\log M_{\odot}\right]} \\
(3)\end{array}$ & $\begin{array}{c}M\left(\mathrm{H}_{2}\right) \\
{\left[\log M_{\odot}\right]} \\
(4)\end{array}$ & $\begin{array}{c}S F R \\
{\left[M_{\odot} \mathrm{yr}^{-1}\right]} \\
(5)\end{array}$ & $\begin{array}{c}\text { Area } \\
{\left[\mathrm{kpc}^{2}\right]} \\
(6)\end{array}$ \\
\hline Haro 11 & 8.20 & 8.70 & 9.40 & 27.6 & 1266 \\
Mrk 1089 & 8.10 & 10.2 & 9.65 & 5.11 & 1350 \\
Mrk 930 & 8.03 & 9.50 & 8.83 & 2.80 & 1617 \\
NGC 4861 & 7.89 & 8.62 & $<8.04$ & 0.048 & 60 \\
NGC 625 & 8.22 & 8.04 & 7.65 & 0.042 & 32 \\
UM 311 & 8.38 & 9.48 & 8.61 & 0.77 & 834 \\
\hline & Additional DGS compact galaxies & \\
\hline Haro 2 & 8.23 & 8.58 & 9.07 & 0.95 & 87 \\
Haro 3 & 8.28 & 9.05 & 8.55 & 0.80 & 65 \\
He 2-10 & 8.43 & 8.49 & 8.52 & 0.68 & 4.8 \\
IZw 18 & 7.14 & 8.00 & $<8.85$ & 0.005 & 1.4 \\
Mrk 209 & 7.74 & 7.43 & 8.47 & 0.005 & 17 \\
NGC 5253 & 8.25 & 8.02 & 7.37 & 0.26 & 23 \\
SBS 0335-052 & 7.25 & 8.64 & $<10.4$ & 0.26 & 3.7 \\
UM 448 & 8.32 & 9.78 & 10.3 & 13.4 & 2.4 \\
UM 461 & 7.73 & 7.86 & $<9.49$ & 0.014 & \\
VII Zw 403 & 7.66 & 7.52 & $<8.79$ & 0.003 & \\
\hline
\end{tabular}

Notes. (1) Galaxy name; (2) metallicities from Madden et al. (2013); (3) aperture-corrected H I masses assuming an exponential distribution, from Rémy-Ruyer et al. (2014); (4) $\mathrm{H}_{2}$ masses using $X_{\mathrm{CO}, Z}$. CO data are from: Israel et al. (1995) for Haro 2; Tacconi \& Young (1987) for Haro 3 and Mrk 209; Kobulnicky et al. (1995) for He 2-10; Leroy et al. (2005) for IZw 18 and VII Zw 403; Taylor et al. (1998) for NGC 5253; Dale et al. (2001) for SBS 0335-052; and Sage et al. (1992) for UM 448 and UM 461. (5) Star formation rate using $L_{\mathrm{TIR}}$ from Rémy-Ruyer et al. (in prep.) and the formula from Murphy et al. (2011); (6) area defined from the Herschel photometry apertures (Rémy-Ruyer et al. 2013) and using distances from Madden et al. (2013).

are $\mathrm{HI}$-dominated and for which the D/G scales more steeply than linearly with metallicity, although its value is strongly variable because it depends on the star formation history of each individual galaxy (Rémy-Ruyer et al. 2014).

To conclude, the use of different methods $\left(X_{\mathrm{CO}, \mathrm{gal}}, X_{\mathrm{CO}, Z}\right.$, or dust) to measure the molecular gas reservoir in our galaxies results in very different mass estimates. This highlights the large uncertainties when using one method or the other. $X_{\mathrm{CO} \text {,gal }}$ gives very low molecular gas masses that probably underestimate the true $\mathrm{H}_{2}$ gas in these low-metallicity galaxies. More realistic molecular masses (accounting for a CO-dark reservoir) are obtained with higher conversion factors from the dust method or from the scaling of $X_{\mathrm{CO}}$ with metallicity.

\subsection{Modeling of the CO emission with RADEX}

We use the non-LTE code RADEX (Van der Tak et al. 2007) to analyze the physical conditions of the low- $J$ CO-emitting gas. RADEX predicts the expected line intensity of a molecular cloud defined by constant column density $\left(N\left({ }^{12} \mathrm{CO}\right)\right)$, gas density $\left(n_{\mathrm{H}_{2}}\right)$, and kinetic temperature $\left(T_{\text {kin }}\right)$. In Fig. 8 , we draw contour plots of CO line ratios for Mrk 1089 (similar plots are obtained for Haro 11, NGC 625, and UM 311) in the $n_{\mathrm{H}_{2}}-T_{\text {kin }}$ space for a given value of $N\left({ }^{12} \mathrm{CO}\right)=3 \times 10^{15} \mathrm{~cm}^{-2}$ (typical of subsolar metallicity clouds; Shetty et al. 2011). The behavior of each line ratio we considered is similar in the $n_{\mathrm{H}_{2}}-T_{\text {kin }}$ space and relatively insensitive to $N\left({ }^{12} \mathrm{CO}\right)$. Given the small number of observational constraints, no precise estimate of the physical conditions of the gas can be reached, except that $T_{\text {kin }} \geq 10 \mathrm{~K}$. To break the degeneracy shown in Fig. 8, and particularly constrain the gas column density better, one would need optically thin line 


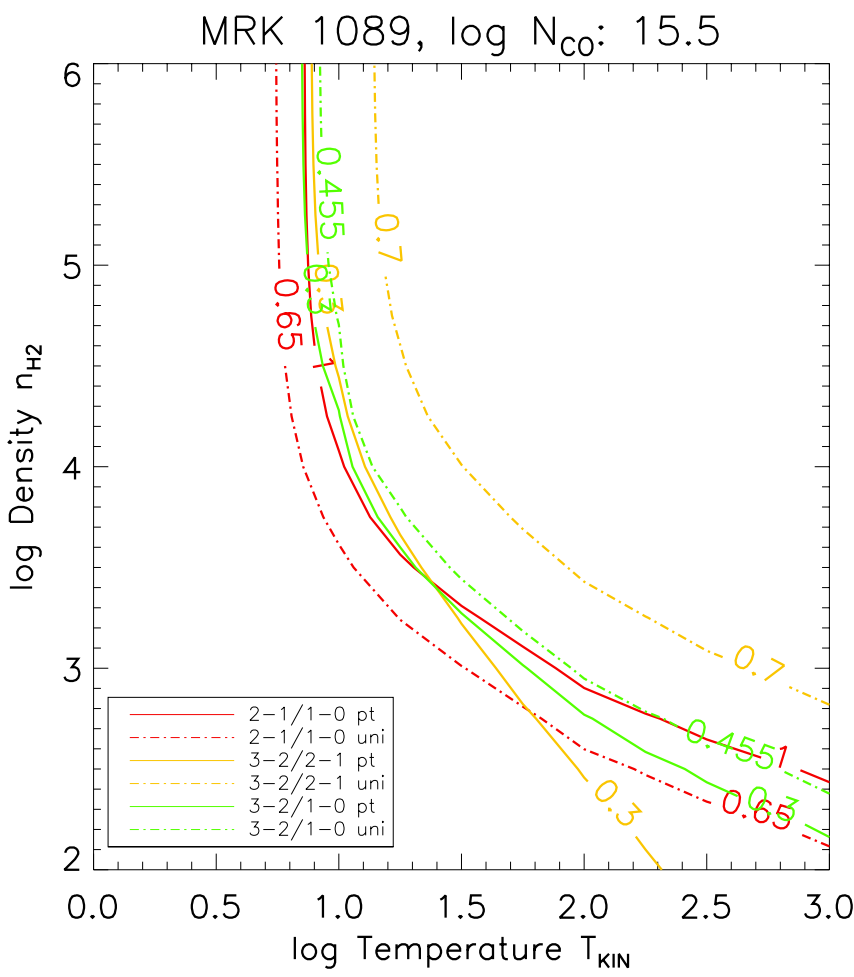

Fig. 8. Results from RADEX for Mrk 1089: contour plot in kinetic temperature $\left(T_{\text {kin }}\right)$-density $\left(n_{\mathrm{H} 2}\right)$ parameter space (logarithmic scale), for a column density of $N\left({ }^{12} \mathrm{CO}\right)=3 \times 10^{15} \mathrm{~cm}^{-2}$. The colored lines correspond to the observed line ratios of $\mathrm{CO}(2-1) / \mathrm{CO}(1-0)$ (red), $\mathrm{CO}(3-2) / \mathrm{CO}(1-0)$ (green), and $\mathrm{CO}(3-2) / \mathrm{CO}(2-1)$ (orange), for both uniform filling (dash-dotted) and point-like (plain) limits.

diagnostics with, e.g., ${ }^{13} \mathrm{CO}$ measurements. When ${ }^{13} \mathrm{CO}$ measurements are available, however, they usually preclude models with only a single molecular gas phase, and require at least two different phases (Meier et al. 2001; Israel 2005).

\subsection{Excitation diagrams of the $\mathrm{H}_{2}$ molecule}

Rotational transitions of molecular hydrogen are visible in the MIR with Spitzer and originate in a warm gas phase (temperature greater than a few hundred $\mathrm{K}$ ), therefore putting constraints on the PDR properties of star-forming regions (and in particular the mass) rather than the cold molecular phase (e.g, Kaufman et al. 2006). As a first step in characterizing the conditions of the PDR, we assume local thermal equilibrium (LTE) and build an excitation diagram to derive the population of the $\mathrm{H}_{2}$ levels and estimate the temperature, column density, and mass of the warm molecular gas. We conduct this analysis only for the two galaxies Haro 11 and NGC 625, which have $\mathrm{H}_{2}$ lines detected in their IRS spectra. We consider the total fluxes in Table 4. The $\mathrm{H}_{2}$ emission is assumed to be optically thin. This approximation is justified by the fact that low-metallicity galaxies have, on average, lower $A_{V}$, and clumpy material of high optical depth, while the warm $\mathrm{H}_{2}$ should arise from regions with larger filling factors. Also, the effects of extinction on the $\mathrm{MIR}_{2}$ lines are not important in normal galaxies (Roussel et al. 2007) and ULIRGs (Higdon et al. 2006). We follow the method from Roussel et al. (2007), and assume a single temperature to match the $S(1)$ to $\mathrm{S}(3)$ lines. This choice is biased toward higher temperatures (and lower masses) due to not detecting the $S(0)$ line, as mentioned in Higdon et al. (2006) and Roussel et al. (2007). The source sizes are taken as the $\mathrm{CO}(1-0)$ Mopra coverage. The excitation
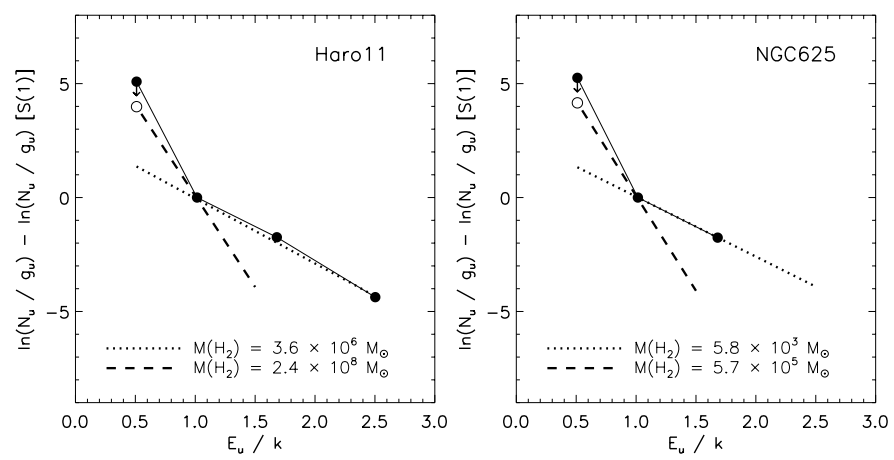

Fig. 9. Excitation diagrams of the $\mathrm{S}(0)$ to $\mathrm{S}(3)$ rotational $\mathrm{H}_{2}$ lines for Haro 11 (left) and NGC 625 (right). The $N_{\mathrm{u}} / g_{\mathrm{u}}$ ratios are normalized by the $\mathrm{S}(1)$ transition. The observations are shown with filled circles. The dotted lines indicate the best fit to the $\mathrm{S}(1)$ to $\mathrm{S}(3)$ transitions. The dashed line indicates the fit to the $\mathbf{S}(0) 1 \sigma$ limit (open circle) and the $\mathrm{S}(1)$ transition.

diagrams are shown in Fig. 9. On the $y$ axis, the observed intensities are converted to column densities in the upper levels $N_{\mathrm{u}}$ normalized by the statistical weights $g_{\mathrm{u}}$.

For Haro 11 (left panel), the best fit to the $S(1), S(2)$, and $\mathrm{S}(3)$ lines agrees with a temperature of $350 \mathrm{~K}$ and column density of $1.1 \times 10^{18} \mathrm{~cm}^{-2}$. The resulting $\mathrm{H}_{2}$ mass is $M\left(\mathrm{H}_{2}\right)>$ $3.6 \times 10^{6} M_{\odot}$, which can be regarded as a lower limit since the $\mathrm{S}(0)$ line is not detected, hence not used as a constraint. Fitting the $1 \sigma$ limit on the $\mathbf{S}(0)$ line and the $\mathbf{S}(1)$ line agrees with a temperature of $100 \mathrm{~K}$ and column density of $7.5 \times 10^{19} \mathrm{~cm}^{-2}$. This yields an upper limit of $M\left(\mathrm{H}_{2}\right)=2.4 \times 10^{8} \mathrm{M}_{\odot}$. The exact warm molecular gas mass is therefore in between the two limits: $3.6 \times 10^{6} M_{\odot}<M\left(\mathrm{H}_{2 \text {,warm }}\right)<2.4 \times 10^{8} \mathrm{M}_{\odot}$. For NGC 625 (right panel), the fit to the $S(1)$ and $S(2)$ lines yields a temperature of $380 \mathrm{~K}$, and a column density of $2.7 \times 10^{17} \mathrm{~cm}^{-2}$. This results in a lower limit on the mass of $M\left(\mathrm{H}_{2}\right)>5.8 \times 10^{3} \mathrm{M}_{\odot}$. Fitting the $1 \sigma$ limit on the $\mathrm{S}(0)$ line and the $\mathrm{S}(1)$ line agrees with a temperature of $120 \mathrm{~K}$ and column density of $2.6 \times 10^{19} \mathrm{~cm}^{-2}$. This gives a warm $\mathrm{H}_{2}$ mass of $M\left(\mathrm{H}_{2}\right)<5.7 \times 10^{5} \mathrm{M}_{\odot}$.

The limits that we obtain on $M\left(\mathrm{H}_{2, \text { warm }}\right)$ indicate that the warm molecular gas mass in Haro 11 and NGC 625 is lower than their cold gas mass (Table 7). Roussel et al. (2007) find an average warm-to-cold ratio of $M\left(\mathrm{H}_{2}\right.$,warm $) / M\left(\mathrm{H}_{2, \text { cold }}\right) \sim 10 \%$ in star-forming galaxies. The temperatures and column densities of the warm $\mathrm{H}_{2}$ gas that we find are generally consistent with previous studies of nearby galaxies. In BCDs, Hunt et al. (2010) find an average temperature for the warm gas of $\sim 245 \mathrm{~K}$, and of $\sim 100 \mathrm{~K}$ for Mrk 996, which is their only galaxy detected in the $\mathrm{S}(0)$ transition. However, their column densities are somewhat higher $\left(N\left(\mathrm{H}_{2}\right) \sim 3 \times 10^{21} \mathrm{~cm}^{-2}\right)$ than ours. In more metalrich galaxies, Roussel et al. (2007) find a median temperature of $\sim 150 \mathrm{~K}$ for the colder $\mathrm{H}_{2}$ component (fitting the $\mathrm{S}(0)$ transition) and a temperature $\geq 400 \mathrm{~K}$ for the warmer component. Their column densities $\left(N\left(\mathrm{H}_{2}\right) \sim 2 \times 10^{20} \mathrm{~cm}^{-2}\right)$ are in better agreement with ours.

\section{Full radiative transfer modeling of Haro 11}

In this section, we investigate a radiative transfer model of Haro 11 using the spectral synthesis code Cloudy v13.01 (Ferland et al. 2013; Abel et al. 2005), for a more complete picture of the conditions in the PDR and molecular cloud. Cormier et al. (2012) analyzed the emission of $\sim 17$ MIR and FIR fine-structure lines from Spitzer and Herschel in Haro 11 
with Cloudy. The model consists of a starburst surrounded by a spherical cloud. It first solves for the photoionized gas, which is constrained by six ionic lines, and then for the PDR, which is constrained by the FIR [O I] lines, assuming pressure equilibrium between the phases. A Galactic grain composition with enhanced abundance of small size grains, as often found in dwarf galaxies, is adopted (see Cormier et al. 2012 for details). We extend this model in light of the molecular data presented.

\subsection{PDR properties}

The PDR model of Cormier et al. (2012) is described by a covering factor $\sim 10 \%$ and parameters $n_{\mathrm{H}}=10^{5.1} \mathrm{~cm}^{-3}$, $G_{0}=10^{3}$ Habing. This model is stopped at a visual extinction $A_{V}$ of 3 mag, just before CO starts to form, to quantify the amount of dark gas untraced by $\mathrm{CO}$, and because no $\mathrm{CO}$ detections had been reported before. The emission of the Spitzer warm $\mathrm{H}_{2}$ lines is produced by this PDR, which has an average temperature of $\sim 200 \mathrm{~K}$. The total gas mass of the PDR derived with the model is $M(\mathrm{PDR})=1.2 \times 10^{8} M_{\odot}$ (Cormier et al. 2012) and is mainly molecular: $90 \%$ is in the form of $\mathrm{H}_{2}$ (dark gas component), and only $10 \%$ in the form of HI. This molecular gas originates in a warm phase, and its mass falls in between the limits on the warm molecular gas mass set in Sect. 4.3. The H I mass of the PDR is lower than the Bergvall et al. (2000) limit, which was the value adopted in Cormier et al. (2012). Thus the higher H I mass found by MacHattie et al. (2014) would not affect our PDR model but rather the diffuse ionized/neutral model component of Cormier et al. (2012).

We find that the intensity of the $\mathrm{H}_{2} \mathrm{~S}(1)$ transition is reproduced by the model within $10 \%$, while the intensities of the $\mathrm{S}(2)$ and $\mathrm{S}(3)$ transitions are underpredicted by a factor of two. The $\mathrm{S}(0)$ prediction is below its upper limit. PDR models indicate that the $\mathrm{H}_{2}$ observations and, particularly, the $\mathrm{S}(2) / \mathrm{S}(1)(0.6)$ and $S(3) / S(1)(0.7)$ ratios could be better matched if another PDR phase of higher density or lower radiation field were considered. However, other factors such as uncertainties in the excitation physics of $\mathrm{H}_{2}$, along with uncertainties in UV and density, can also play a role (Kaufman et al. 2006). Reproducing lines coming from $J>3$ have inherent uncertainties in the microphysics, and inconsistencies can arise from trying to simultaneously reproduce these lines at the same time as reproducing [C II] and $[\mathrm{OI}]$.

\subsection{Molecular cloud properties}

To model the molecular cloud $\left(A_{V} \geq 3 \mathrm{mag}\right.$, where CO starts to form) with Cloudy, we continue the PDR model described above to larger $A_{V}$. This way, we assume that the observed $\mathrm{CO}$ emission is linked to the densest star-forming cores rather than to a diffuse phase. Since we have no observations of molecular optically thin tracers or denser gas tracers than $\mathrm{CO}$, we use the cold dust emission to set our stopping criterion for the models and determine the $A_{V}$ appropriate to reproduce the FIR/submm emission of the spectral energy distribution of Haro 11. We find that the Herschel PACS and SPIRE photometry is best reproduced (within $30 \%$ ) for $A_{V} \sim 30 \mathrm{mag}$, and we take this value as our stopping criterion.

We find that the $\mathrm{CO}$ emission is essentially produced at $3<A_{V}<6 \mathrm{mag}$, where CO dominates the cooling of the gas. For larger $A_{V}$, the gas temperature stabilizes around $30 \mathrm{~K}$, and denser gas tracers such as $\mathrm{HNC}, \mathrm{CN}$, and CS take over the cooling. Densities in the molecular cloud reach $10^{6.3} \mathrm{~cm}^{-3}$. For
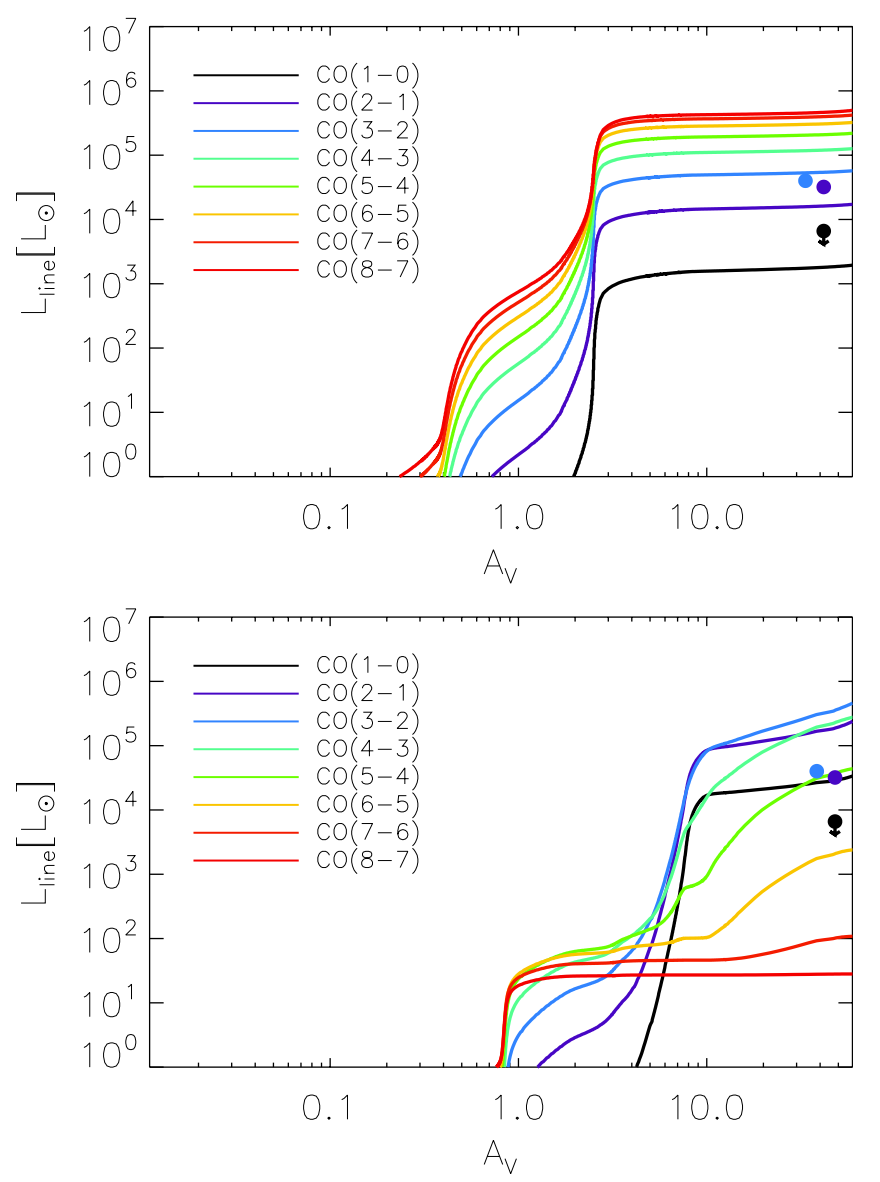

Fig. 10. Cloudy model predictions for the COline luminosities as a function of $A_{V}$. The models are stopped at $A_{V}$ of $30 \mathrm{mag}$, and the covering factor is set to $\sim 5 \%$. Observations of the $\mathrm{CO}(1-0)$ (upper limit), $\mathrm{CO}(2-1)$, and $\mathrm{CO}(3-2)$ lines are represented by filled circles on the right-hand side of the plots. Top: no turbulent velocity included. Bottom: including a turbulent velocity $u_{\text {turb }}$ of $30 \mathrm{~km} \mathrm{~s}^{-1}$.

$A_{V}>10 \mathrm{mag}, \sim 30 \%$ of the carbon is in the form of CO, and the condensation of CO onto grain surfaces reaches $\sim 10 \%$. The photoelectric effect on grains is mostly responsible for the heating of the gas at the surface of the cloud, and grain collisions are the main heating source deeper into the cloud. Figure 10 (top) shows the Cloudy model predictions for the CO line luminosities (from $\mathrm{CO}(1-0)$ to $\mathrm{CO}(8-7)$ ) as a function of $A_{V}$ (models are stopped at $\left.A_{V}=30 \mathrm{mag}\right)$. The observed $\mathrm{CO}(2-1)$ and $\mathrm{CO}(3-2)$ luminosities are matched by this model within a factor of 2 , and the $\mathrm{CO}(1-0)$ model prediction falls below the observed upper limit. The predicted $\mathrm{CO}(1-0)$ intensity is only three times lower than our observed upper limit, indicating that higherperformance telescopes (such as ALMA) should be able to detect this transition. The $\mathrm{CO}(3-2) / \mathrm{CO}(2-1)$ ratio is overpredicted by a factor of 3 compared to the observed uniform-filling ratio.

The modeled total mass depends strongly on the assumed geometry and on the SED fitting, which has an additional complication due to an excess of submm emission (Galametz et al. 2011). We select two values of maximum $A_{V}$, one at which the shielding is sufficient for CO to completely form and the SED is well fit (within $30 \%)$ up to $350 \mu \mathrm{m}\left(A_{V, \max 1}=15 \mathrm{mag}\right.$ ), and one at which the SED is fit up to $500 \mu \mathrm{m}\left(A_{V, \max 2}=30 \mathrm{mag}\right)$. The molecular gas masses corresponding to those $A_{V}$ are $M\left(\mathrm{H}_{2}\right)=$ $7.2 \times 10^{8} M_{\odot}$ and $M\left(\mathrm{H}_{2}\right)=1.6 \times 10^{9} M_{\odot}$, respectively. We adopt the latter value in the rest of this paper, but recall that this mass estimate is uncertain by a factor of $\sim 2$. This is the mass in 
the molecular phase associated with $\mathrm{CO}$ emission. The molecular gas mass in the CO-dark PDR layer is then $\sim 10 \%$ that of the CO-emitting core (Sect. 5.1), so the warm-to-cold molecular gas mass in Haro 11 is very close to the values found in Roussel et al. (2007). The total molecular mass in Haro 11 (CO-dark gas $+\mathrm{CO}$ core) is $1.7 \times 10^{9} M_{\odot}$, close to the value derived from $X_{\mathrm{CO}, Z}$. The resulting $\alpha_{\mathrm{CO} \text {,model }}$ is $\sim 50 M_{\odot}\left(\mathrm{K} \mathrm{km} \mathrm{s}^{-1} \mathrm{pc}^{2}\right)^{-1}$ and is about ten times $\alpha_{\mathrm{CO}, \text { gal }}=4.3 M_{\odot}\left(\mathrm{K} \mathrm{km} \mathrm{s}^{-1} \mathrm{pc}^{2}\right)^{-1}$. The total $\left(\mathrm{H} \mathrm{II}+\mathrm{H} \mathrm{I}+\mathrm{H}_{2}\right.$, including $\mathrm{He}$ ) model gas mass is $3.3 \times 10^{9} \mathrm{M}_{\odot}$, and the total gas mass to stellar mass $\left(10^{10} M_{\odot}\right)$ ratio is $\sim 35 \%$. Despite active photodissociation and low CO luminosities, the overall molecular gas content of Haro 11 is not particularly low. The mass budget of Haro 11 is dominated by its molecular gas, followed by the ionized and neutral gas phases.

Modeling the molecular cloud to $A_{V}$ of 30 mag has implications on the model predictions for the lines coming from other media given the geometry adopted for the model. Even though the ionic lines are affected little, the PDR $\mathrm{H}_{2}$ lines can be subject to dust extinction, and the [O I] lines become optically thick, their emission being reduced by a factor of two at most. If true, then we would need an additional component in the model, another PDR phase or other excitation mechanisms, such as shocks (e.g., Hollenbach \& McKee 1989) to reproduce the observations. Spatially resolved observations of, say, [C I] for the PDR, as well as optically thin molecular tracers, are needed to confirm this PDR/molecular structure.

\subsection{Influence of micro-turbulence}

We discuss the effects of adding a turbulent velocity in the Cloudy models, in particular to the predicted $\mathrm{CO}$ line ratios, which are sensitive to the temperature in the high-density regime. Turbulence may be an important factor in setting the ISM conditions in Haro 11 since it has been found to be dynamically unrelaxed (Östlin et al. 2001; James et al. 2013). The microphysical turbulence is linked to the observed CO FWHM $\left(\sim 60 \mathrm{~km} \mathrm{~s}^{-1}\right)$ via $u_{\mathrm{obs}}=\sqrt{u_{\mathrm{thermal}}^{2}+u_{\mathrm{turb}}^{2}}=F W H M / \sqrt{4 \ln (2)}$. Turbulence is included in the pressure balance, with the term $P_{\text {turb }} \propto n_{\mathrm{H}} u_{\text {turb }}^{2}$, hence dissipating some energy away from the cloud.

We show the temperature profile in the cloud in Fig. 11 (top panel). We have chosen to display the results for a turbulent velocity of $30 \mathrm{~km} \mathrm{~s}^{-1}$ to illustrate the effect of turbulence on the profiles clearly. The gas temperature decreases more quickly with $A_{V}$ in the molecular cloud from $200 \mathrm{~K}$ down to $7 \mathrm{~K}$, and stabilizes around $10 \mathrm{~K}$ in the $\mathrm{CO}$-emitting region. The density stays around that of the PDR $\left(10^{5.2} \mathrm{~cm}^{-3}\right)$, so similar results are found in the case of a constant density. Adding turbulence has a profound effect on the CO ladder, where high- $J$ lines are emitted in a thinner region close to the cloud surface compared to low- $J$ CO lines (Fig. 10, bottom). With turbulence, the low- $J$ CO lines start to form at $A_{V}$ of 5-6 mag. The [C I] 370 and $610 \mu \mathrm{m}$ lines are actually enhanced by an order of magnitude between $A_{V}$ of 2-5 mag, and then the lowest $\mathrm{CO}$ transitions (lowest $T_{\text {exc }}$ ) are enhanced. As a consequence, ratios of $\mathrm{CO}(3-2) / \mathrm{CO}(2-1)$ decrease with increasing turbulent velocity (Fig. 11, bottom). The observed $\mathrm{CO}(3-2) / \mathrm{CO}(2-1)$ ratio is matched better by the models when including a turbulent velocity $>10 \mathrm{~km} \mathrm{~s}^{-1}$. Observations of $[\mathrm{CI}]$ and higher- $J(J>4) \mathrm{CO}$ lines would help validate the importance of turbulence and investigate possible excitation mechanisms in low-metallicity galaxies.
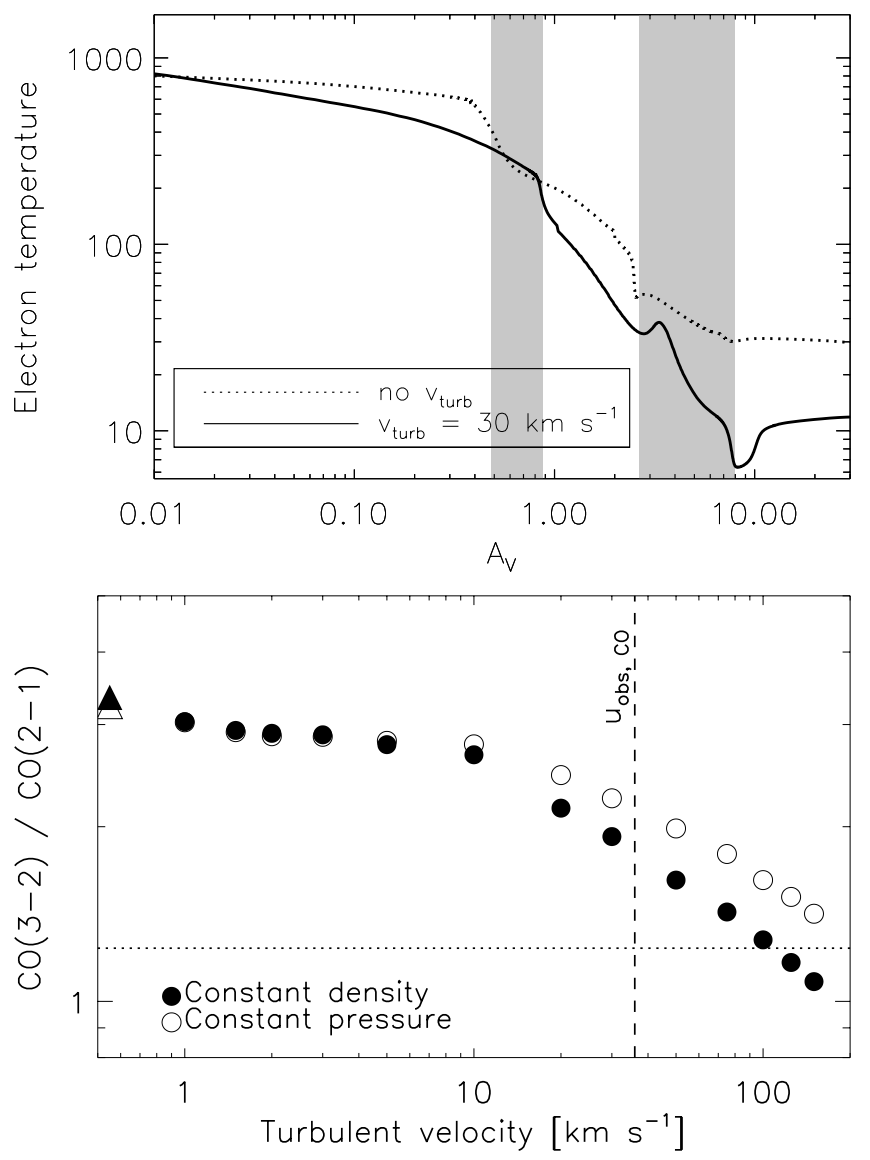

Fig. 11. Top: temperature profile in the cloud. The dotted profile is without turbulence, and the plain profile with a turbulent velocity of $30 \mathrm{~km} \mathrm{~s}^{-1}$. The first vertical gray band indicates the transition into the molecular cloud $\left(\mathrm{H}_{2}>\mathrm{HI}\right)$, and the second gray band when $\mathrm{CO}$ starts to form. Bottom: cloudy model predictions for the $\mathrm{CO}(3-2) / \mathrm{CO}(2-1)$ ratio as a function of turbulent velocity. The two cases of constant density (open symbols) and constant pressure (filled symbols) are considered. The horizontal dotted line indicates the observed $\mathrm{CO}$ ratio and the vertical dashed line the observed CO velocity $u_{\mathrm{obs}}=F W H M / \sqrt{4 \ln (2)}$. Triangles correspond to the model without turbulent velocity.

\section{Star formation and total gas reservoir}

In this section, we analyze the global star formation properties of our low-metallicity galaxies and relate their gas reservoir to the star formation activity in order to contrast what we find for SFE when we use a tracer of a specific ISM phase as a proxy for the total gas reservoir.

\section{1. $[\mathrm{CII}],[\mathrm{OI}]$, and $\mathrm{CO}$}

The $[\mathrm{C}$ II $]-$ to- $\mathrm{CO}(1-0)$ ratio can be used as a diagnostic of the star formation activity in nearby normal galaxies (Stacey et al. 1991) and high-redshift galaxies $(z \sim 1-2$; Stacey et al. 2010), with average values between 2000 and 6000 (for star-forming galaxies). High [C II]/CO ratios are observed in low-metallicity dwarf galaxies (Madden 2000) because of the different cloud structure with smaller CO cores and larger PDR envelopes, and also because of increased photoelectric heating efficiencies in the PDR, caused by dilution of the UV radiation field that can enhance the [C II] cooling (Israel \& Maloney 2011). For our sample, we also find high ratios with $[\mathrm{C} \mathrm{II}] / \mathrm{CO}(1-0) \geq 7000$ (Fig. 12, top left). This indicate that most of the gas is exposed to intense 

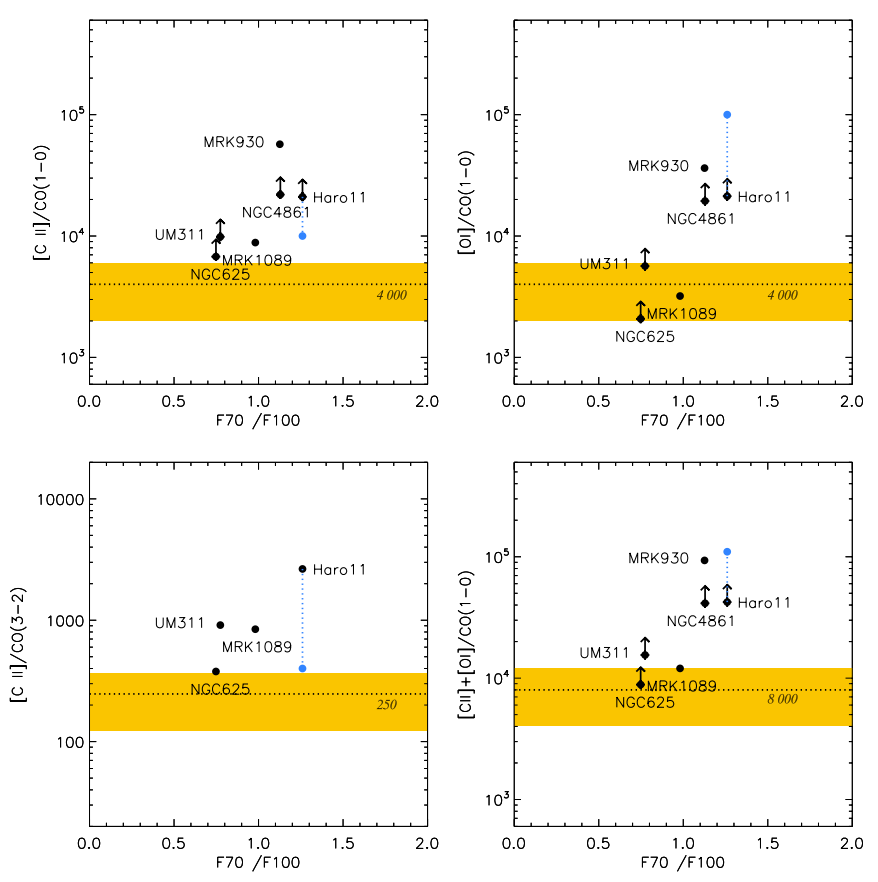

Fig. 12. [C II] and [O I]-to-CO ratios versus $70 \mu \mathrm{m} / 100 \mu \mathrm{m}$. The horizontal orange band indicates the average ratio [C II $] / C O(1-0)$ of $2000-$ 6000 observed in star-forming regions of the Galaxy and galaxies (Stacey et al. 1991). For the $[\mathrm{C} \mathrm{II}] / \mathrm{CO}(3-2)$ horizontal band, a ratio $R_{31}=0.6$ is considered. The blue circles correspond to the model predictions for Haro 11.

radiation fields and that the star-forming regions dominate the global emission of our galaxies.

$\mathrm{C}^{0}$ has an ionization potential of $11.26 \mathrm{eV}$, and critical densities of $50 \mathrm{~cm}^{-3}$ for collisions with electrons and of $3 \times 10^{3} \mathrm{~cm}^{-3}$ for collisions with hydrogen atoms. Thus, $\mathrm{C}^{+}$can arise from the surface layers of PDRs but also from diffuse ionized gas, as well as diffuse neutral gas. In Fig. 12, we also show line ratios with [OI], which could be a more accurate tracer of the PDR envelope given the possible origins of the [C II] emission, and with $\mathrm{CO}(3-2)$, for which we have more detections than $\mathrm{CO}(1-0)$. The $[\mathrm{OI}] / \mathrm{CO}(1-0)$ ratios are generally lower than the $[\mathrm{C}$ II $] / \mathrm{CO}(1-0)$ ratios because of the high $[\mathrm{C} \mathrm{II}] /[\mathrm{O} \mathrm{I}]$ ratios found in our dwarf galaxies. However, the ratios remain high when using [O I]. Although there are only four data points, the scatter in the $[\mathrm{C} \mathrm{II}] / \mathrm{CO}(3-2)$ values is slightly reduced. The color ratio of $70 \mu \mathrm{m} / 100 \mu \mathrm{m}$ is an indicator of the dust temperature and thus of the relative contribution of the H II region and PDR to the global emission. One might, therefore, expect higher line-to-CO ratios for higher $70 \mu \mathrm{m} / 100 \mu \mathrm{m}$ ratios. We do not see a direct correlation between the observed line-to-CO ratios and $70 \mu \mathrm{m} / 100 \mu \mathrm{m}$ color or metallicity, but maybe a slight increase of $[\mathrm{C} \mathrm{II}] / \mathrm{CO}$ with $70 \mu \mathrm{m} / 100 \mu \mathrm{m}$.

\subsection{Star formation law}

We investigate how the different gas reservoirs of $\mathrm{HI}$ and $\mathrm{H}_{2}$ are related to the star formation activity measured in our galaxies, and compare them to the dataset of starburst and spiral galaxies from Kennicutt (1998), as well as the other compact dwarf galaxies from the DGS with $\mathrm{CO}(1-0)$ measurements. We look at the SFR surface density $\left(\Sigma_{\mathrm{SFR}}\right)$ and gas surface density $\Sigma_{\text {gas }}=\Sigma_{\mathrm{HI}}+\Sigma_{\mathrm{H}_{2}}$. For $\Sigma_{\mathrm{SFR}}$, we use the $L_{\mathrm{TIR}}$-derived SFRs from the formula of Murphy et al. (2011), divided by the source

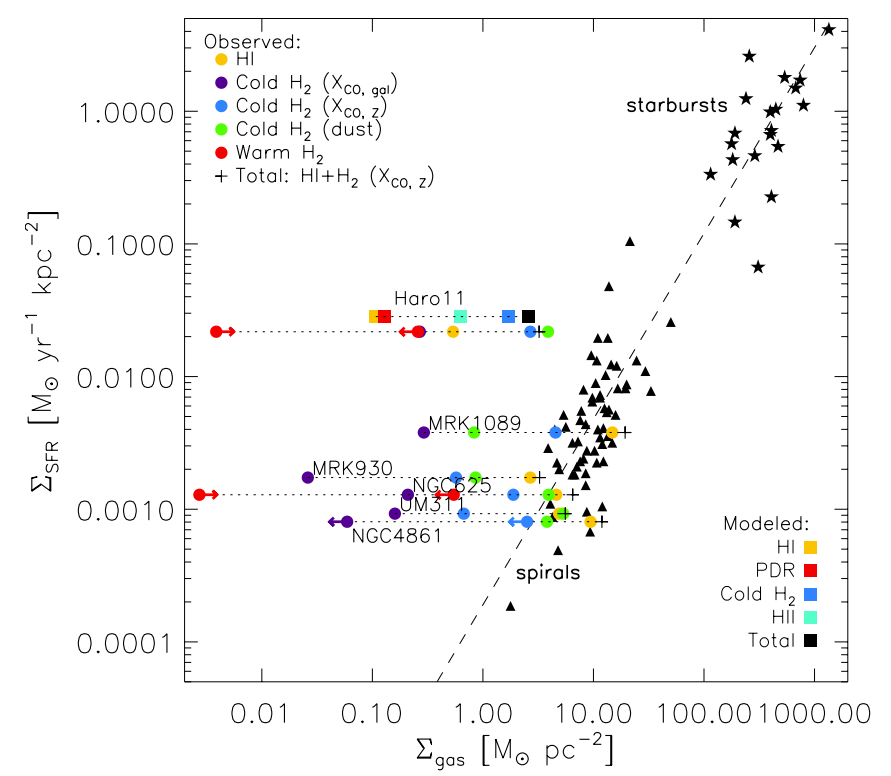

Fig. 13. Star formation rate surface density $\left(\Sigma_{\mathrm{SFR}}\right)$ versus gas surface density $\left(\Sigma_{\text {gas }}\right)$. Our dwarf galaxies are represented by filled circles, and the colors show the contribution from the separate observed phases. Cloudy models for Haro 11 correspond to the filled rectangles (Sect. 5.2). The starbursts and spirals from Kennicutt (1998) are represented by stars and triangles, respectively. The diagonal dotted line indicates a fit to the Schmidt-Kennicutt law, with power index 1.4 to starbursts and spirals (Kennicutt 1998).

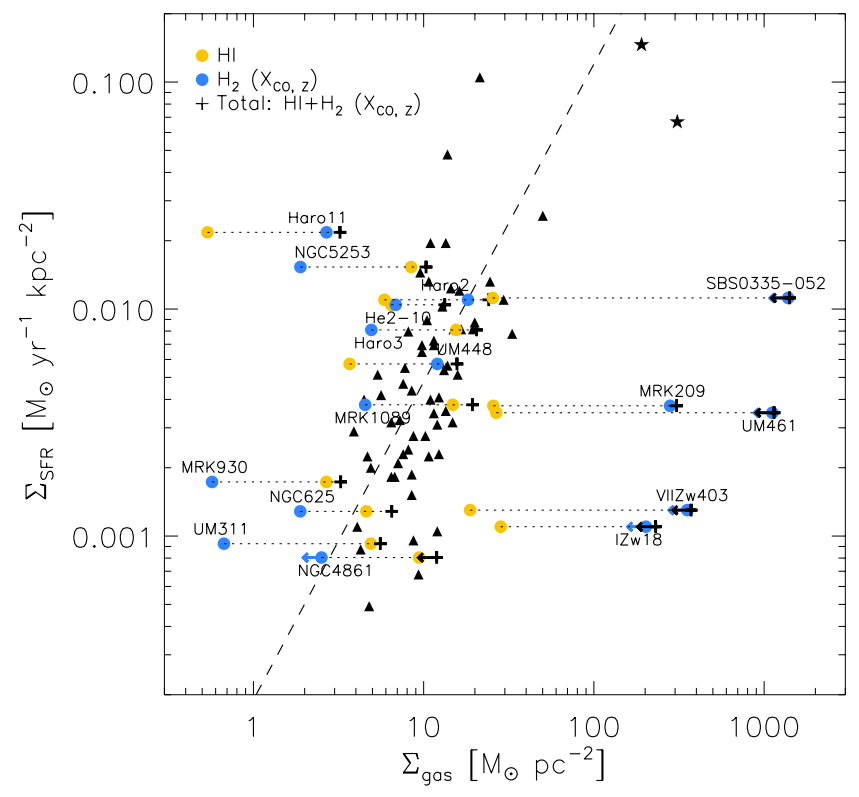

Fig. 14. Same as Fig. 13 for all the compact dwarf galaxies of the DGS with $\mathrm{CO}$ observations.

size, which is taken as the dust aperture from Rémy-Ruyer et al. (2013). For the hydrogen masses, we consider the Hi within the dust apertures from Rémy-Ruyer et al. (2014), and the $\mathrm{H}_{2}$ derived from total $\mathrm{CO}$ intensities with $X_{\mathrm{CO}, \text { gal }}, X_{\mathrm{CO}, Z}$, and from dust measurements (Table 7) for our sample (Fig. 13), and with $X_{\mathrm{CO}, Z}$ and CO intensities from the literature for the DGS galaxies (Fig. 14). Those galaxies have metallicities between $1 / 40$ and $1 Z_{\odot}$ (Madden et al. 2013). We summarize those parameters in Table 9 . The hydrogen masses are multiplied by a factor 1.36 in the figures to include helium. 
In Figs. 13 and 14, we see that most low-metallicity galaxies fall close to the Schmidt-Kennicutt relation. The less active galaxies are generally dominated by their H I gas, while some of the most active galaxies, such as Haro 11, are dominated by their $\mathrm{H}_{2}$ gas. Several galaxies stand farther to the right of the relation (IZw 18, Mrk 209, NGC 4861, UM 461, VII Zw 403), hence appear either more gas-rich, with part of their H I gas probably less connected to the star formation activity, or more quiescent than expected, where the TIR luminosity might slightly underestimate the star formation rate (Kennicutt \& Evans 2012). Mrk 209 shows a strikingly high molecular surface density, which would be an indication that it is on the verge of forming stars. However, it is known to already host young super star clusters and WR features (e.g., Thuan \& Izotov 2005). Moreover, its warm molecular component inferred from Spitzer data is only $\sim 6 \times 10^{3} M_{\odot}$ (Hunt et al. 2010), which is orders of magnitude lower than the estimated cold molecular gas. These indications combined probably mean that we are overestimating $\Sigma_{\mathrm{H}_{2}}$ with the high conversion factor $X_{\mathrm{CO}, Z}$ used (and because of uncertainties on the metallicity). Two galaxies (Haro 11 and, more moderately, NGC 5253), which have the highest $\Sigma_{\mathrm{SFR}}$, lie to the left of the standard Schmidt-Kennicutt relation, even when considering high $X_{\mathrm{CO}}$ values. Both are active blue compact galaxies. The presence of young super star clusters in NGC 5253 and clear lack of CO suggest that the starburst was triggered by accretion of gas from outside the galaxy and formed with high efficiency (Meier et al. 2002). Haro 11 is also known to have undergone a merger event (Östlin et al. 1999) and behaves like starbursting mergers (Daddi et al. 2010). We note that the position of our low-metallicity galaxies coincide with the position of spiral galaxies on Fig. 14. We have used apertures that are large enough to encompass the total FIR continuum emission and are $\geq 1.5 \times r_{25}$ (Rémy-Ruyer et al. 2013). If we were to zoom on the star-forming regions of our galaxies, those would generally move closer towards the position of the starburst galaxies.

The gas depletion time, i.e. the time needed to consume the existing gas reservoir given the SFR, is expressed as the inverse of the star formation efficiency: $\tau_{\text {dep }}=\Sigma_{\text {gas }} / \Sigma_{\text {SFR }}$ [yr]. In nearby, predominantly late-type disk galaxies, the average molecular depletion time found by Bigiel et al. (2008) is around 2 Gyr. For our sample, taking the cold $\mathrm{H}_{2}\left(X_{\mathrm{CO}, Z}\right)$ mass values from Table 7 and including helium, we get molecular depletion times of $~ 0.1 \mathrm{Gyr}$ (for Haro 11) - 0.3 Gyr (for Mrk 930) up to $3 \mathrm{Gyr}$ (for NGC 4861), and a median value of $0.7 \mathrm{Gyr}$ for the compact DGS sample. Sage et al. (1992) find similarly low values $(\sim 0.1 \mathrm{Gyr})$ for their sample of BCDs, although they use relatively lower conversion factors, of two to three times $X_{\mathrm{CO} \text {,gal }}$. Therefore, even when using a metallicity-scaled $X_{\mathrm{CO}}$ factor, we find that our systems have short molecular gas depletion times typical of interacting systems, starbursts, and ULIRGs.

If we are accounting for all the gas related to the star formation, such short depletion times indicate that our dwarf galaxies are efficient in forming stars. This can be understood by the fact that several super star clusters are observed in those galaxies, and BCGs do have higher cluster formation efficiencies than normal galaxies (Adamo et al. 2011a). If these galaxies keep forming stars at the current rate, they would consume all their gas quickly. Therefore they might be caught in a bursty episode (since they are selected as bright in the IR). When adding the H I data, the average total gas depletion time over the sample is $\tau_{\text {dep }} \sim 5$ Gyr. This value is significantly higher than the molecular depletion times since most galaxies are H I-dominated. This H I gas may also play a role in regulating the star formation, as direct fuel at very low metallicities (Krumholz 2012) or as future fuel, since part of the H I we measure may be residing in the envelopes of the molecular clouds. Regardless of the role of the H I, the picture that emerges is a very rapid and efficient conversion of molecular gas into stars in low-metallicity dwarf galaxies.

\section{Conclusions}

The state and abundance of the molecular gas, observed through the molecule $\mathrm{CO}$, is not well known in dwarf galaxies. To address this issue, we obtained new observations of the $\mathrm{CO}(1-0), \mathrm{CO}(2-1)$, and $\mathrm{CO}(3-2)$ lines in a subsample of six low-metallicity galaxies of the Herschel Dwarf Galaxy Survey (Haro 11, Mrk 930, Mrk 1089, NGC 4861, NGC 625, and UM 311), which we combine with existing broad band and atomic fine-structure line observations. We summarize our results as follows.

- Out of the six targets, five are detected in at least one CO transition, but NGC 4861 remains undetected in CO. Three of the targets, Haro 11, Mrk 930 (marginal detection), and UM 311, with metallicities $Z \sim 1 / 3,1 / 5$, and $1 / 2 Z_{\odot}$ respectively, were never detected in $\mathrm{CO}$ before, and no $\mathrm{CO}$ data were reported in the literature for NGC 625.

- $\mathrm{CO}$ is found to be faint in these six dwarf galaxies, especially relative to the [C II] $157 \mu \mathrm{m}$ line, since we derive $[\mathrm{C}$ II $] / C O(1-0)$ ratios $\geq 7000$. In particular, this hints at the low filling factor of the $\mathrm{CO}$-emitting material and high photoelectric [C II] heating efficiency, as previously suggested (Israel et al. 1996; Madden 2000).

- Molecular gas mass estimates are quite uncertain and vary significantly depending on the method used $\left(X_{\mathrm{CO}}\right.$, dust measurements, etc.). A Galactic conversion factor predicts unrealistically low masses, while the dust method, a metallicityscaled $X_{\mathrm{CO}}$, or radiative transfer modeling predict higher molecular masses with low accuracy. Even when considering high $X_{\mathrm{CO}}$ conversion factors for our sample of lowmetallicity galaxies, the molecular content makes a small contribution to the total ISM mass budget compared to the H I gas (except for Haro 11).

- We compared the star formation activity to the gas reservoir for a sample extended to the compact low-metallicity galaxies of the DGS. Despite their low molecular content, all galaxies are in relative agreement with the (total gas) Schmidt-Kennicutt relation. The BCGs NGC 5253 and Haro 11, which has a high molecular-to-atomic gas fraction, are exceptions and may be intrinsically more efficient at forming stars. Even with a metallicity-scaled $X_{\mathrm{CO}}$ factor, the molecular gas depletion times of our low-metallicity galaxies are short. Our results imply either that 1) the molecular gas forms stars with a different efficiency in the dwarf galaxies of our sample than in normal spirals or 2) if a universal total (as opposed to molecular) gas depletion time applies, then the atomic and possibly the ionized gas are participating in star formation in those galaxies.

What the role of the different ISM gas phases is and whether star formation efficiencies are truly higher in some dwarf galaxies is still unclear. Our interpretations are limited by the different antenna beam sizes and the lack of information on the source size and distribution. In particular, we cannot conclude whether the cold ISM in the dwarf galaxies that we have studied is a result of enhanced photodissociation of molecular clouds having a mass distribution similar to clouds in normal metallicity galaxies, or if it points to a fundamentally altered structure of the lowmetallicity ISM with molecular clouds having a different mass 
distribution compared to $\mathrm{H}_{2}$ clouds in normal metallicity galaxies. The analysis presented here could be taken to the next level with interferometry observations with the SMA, ALMA, or the JVLA to map the distribution and properties of the gas reservoirs on smaller scales.

Acknowledgements. This research was supported by Sonderforschungsbereich SFB 881 "The Milky Way System" of the German Research Foundation (DFG), and by the Agence Nationale de la Recherche (ANR, France) through the programme SYMPATICO (Programme Blanc Projet ANR-11-BS56-0023). V.L. is supported by a CEA/Marie Curie Eurotalents fellowship. This research made use of the NASA/IPAC Extragalactic Database (NED), which is operated by the Jet Propulsion Laboratory, California Institute of Technology, under contract with the National Aeronautics and Space Administration. This work is based in part on observations made with the Spitzer Space Telescope, which is operated by the Jet Propulsion Laboratory, California Institute of Technology under a contract with NASA. The Mopra Radio Telescope is part of the Australia Telescope National Facility, which is funded by the Commonwealth of Australia for operation as a National Facility managed by CSIRO. The University of New South Wales Digital Filter Bank used for the observations with the Mopra Telescope was provided with support from the Australian Research Council. We thank the IRAM-30 m staff for their help with the observations. This publication is based on data acquired with the Atacama Pathfinder Experiment (APEX). APEX is a collaboration between the Max-Planck-Institut für Radioastronomie, the European Southern Observatory, and the Onsala Space Observatory.

\section{References}

Abel, N. P., Ferland, G. J., Shaw, G., \& van Hoof, P. A. M. 2005, ApJS, 161, 65 Ackermann, M., Ajello, M., Baldini, L., et al. 2011, ApJ, 726, 81

Adamo, A., Östlin, G., \& Zackrisson, E. 2011a, MNRAS, 417, 1904

Adamo, A., Östlin, G., Zackrisson, E., et al. 2011b, MNRAS, 415, 2388

Albrecht, M., Chini, R., Krügel, E., Müller, S. A. H., \& Lemke, R. 2004, A\&A, 414,141

Asplund, M., Grevesse, N., Sauval, A. J., \& Scott, P. 2009, ARA\&A, 47, 481

Bayet, E., Gerin, M., Phillips, T. G., \& Contursi, A. 2004, A\&A, 427, 45

Bendo, G. J., Galliano, F., \& Madden, S. C. 2012, MNRAS, 423, 197

Bergvall, N., Masegosa, J., Östlin, G., \& Cernicharo, J. 2000, A\&A, 359, 41

Bigiel, F., Leroy, A., Walter, F., et al. 2008, AJ, 136, 2846

Bigiel, F., Leroy, A. K., Walter, F., et al. 2011, ApJ, 730, L13

Bolatto, A. D., Jackson, J. M., Israel, F. P., Zhang, X., \& Kim, S. 2000, ApJ, 545, 234

Bolatto, A. D., Leroy, A., Israel, F. P., \& Jackson, J. M. 2003, ApJ, 595, 167

Bolatto, A. D., Wolfire, M., \& Leroy, A. K. 2013, ARA\&A, 51, 207

Braine, J., Duc, P.-A., Lisenfeld, U., et al. 2001, A\&A, 378, 51

Cannon, J. M. 2004, Ph.D. Thesis, University of Minnesota, USA

Cannon, J. M., McClure-Griffiths, N. M., Skillman, E. D., \& Côté, S. 2004, ApJ, 607,274

Cannon, J. M., Skillman, E. D., Sembach, K. R., \& Bomans, D. J. 2005, ApJ, 618,247

Cormier, D., Madden, S. C., Hony, S., et al. 2010, A\&A, 518, L57

Cormier, D., Lebouteiller, V., Madden, S. C., et al. 2012, A\&A, 548, A20

Daddi, E., Elbaz, D., Walter, F., et al. 2010, ApJ, 714, L118

Dale, D. A., Helou, G., Contursi, A., Silbermann, N. A., \& Kolhatkar, S. 2001, ApJ, 549, 215

Dame, T. M., Hartmann, D., \& Thaddeus, P. 2001, ApJ, 547, 792

de Vaucouleurs, G., de Vaucouleurs, A., Corwin, Jr., H. G., et al. 1991, Third Reference Catalogue of Bright Galaxies, Volume I: Explanations and references, Volume II: Data for galaxies between $0^{\mathrm{h}}$ and $12^{\mathrm{h}}$, Volume III: Data for galaxies between $12^{\mathrm{h}}$ and $24^{\mathrm{h}}$ (New York: Springer Verlag)

Edmunds, M. G. 2001, MNRAS, 328, 223

Elmegreen, B. G., Rubio, M., Hunter, D. A., et al. 2013, Nature, 495, 487

Ferland, G. J., Porter, R. L., van Hoof, P. A. M., et al. 2013, Rev. Mex. Astron. Astrofis., 49, 137

Galametz, M., Madden, S., Galliano, F., et al. 2009, A\&A, 508, 645 Galametz, M., Madden, S. C., Galliano, F., et al. 2011, A\&A, 532, A56

Galliano, F., Hony, S., Bernard, J.-P., et al. 2011, A\&A, 536, A88

Genzel, R., Tacconi, L. J., Combes, F., et al. 2012, ApJ, 746, 69

Gil de Paz, A., Boissier, S., Madore, B. F., et al. 2007, ApJS, 173, 185

Glover, S. C. O., \& Clark, P. C. 2012, MNRAS, 421, 9

Glover, S. C. O., Federrath, C., Mac Low, M.-M., \& Klessen, R. S. 2010, MNRAS, 404, 2

Grenier, I. A., Casandjian, J.-M., \& Terrier, R. 2005, Science, 307, 1292

Grimes, J. P., Heckman, T., Strickland, D., et al. 2007, ApJ, 668, 891

Guseva, N. G., Izotov, Y. I., \& Thuan, T. X. 1998, Kinem. Phys. Celest. Bodies, 14,41
Hickson, P. 1982, ApJ, 255, 382

Higdon, S. J. U., Devost, D., Higdon, J. L., et al. 2004, PASP, 116, 975

Higdon, S. J. U., Armus, L., Higdon, J. L., Soifer, B. T., \& Spoon, H. W. W. 2006, ApJ, 648, 323

Hollenbach, D., \& McKee, C. F. 1989, ApJ, 342, 306

Hopkins, A. M., Schulte-Ladbeck, R. E., \& Drozdovsky, I. O. 2002, AJ, 124, 862

Houck, J. R., Roellig, T. L., Van Cleve, J., et al. 2004, in SPIE Proc. 5487, ed. J. C. Mather, 62

Hunt, L. K., Thuan, T. X., Izotov, Y. I., \& Sauvage, M. 2010, ApJ, 712, 164

Iglesias-Paramo, J., \& Vilchez, J. M. 1997, ApJ, 479, 190

Indebetouw, R., Brogan, C., Chen, C.-H. R., et al. 2013, ApJ, 774, 73

Israel, F. 2000, in Molecular Hydrogen in Space, eds. F. Combes \& G. Pineau

des Fôrets (Cambridge: Cambridge University Press), 293

Israel, F. P. 2005, A\&A, 438, 855

Israel, F. P., \& Maloney, P. R. 2011, A\&A, 531, A19

Israel, F. P., Tacconi, L. J., \& Baas, F. 1995, A\&A, 295, 599

Israel, F. P., Maloney, P. R., Geis, N., et al. 1996, ApJ, 465, 738

Izotov, Y. I., \& Thuan, T. X. 1998, ApJ, 500, 188

James, B. L., Tsamis, Y. G., Walsh, J. R., Barlow, M. J., \& Westmoquette, M. S. 2013, MNRAS, 430, 2097

Kaufman, M. J., Wolfire, M. G., \& Hollenbach, D. J. 2006, ApJ, 644, 283

Kennicutt, Jr., R. C. 1998, ApJ, 498, 541

Kennicutt, R. C., \& Evans, N. J. 2012, ARA\&A, 50, 531

Kennicutt, Jr., R. C., Lee, J. C., Funes, J. G., S. J., Sakai, S., \& Akiyama, S. 2008, ApJS, 178, 247

Kester, D. J. M., Beintema, D. A., \& Lutz, D. 2003, in The Calibration Legacy of the ISO Mission, eds. L. Metcalfe, A. Salama, S. B. Peschke, \& M. F. Kessler, ESA SP, 481, 375

Kobulnicky, H. A., Dickey, J. M., Sargent, A. I., Hogg, D. E., \& Conti, P. S. 1995, AJ, 110, 116

Krumholz, M. R. 2012, ApJ, 759, 9

Krumholz, M. R., Leroy, A. K., \& McKee, C. F. 2011, ApJ, 731, 25

Ladd, N., Purcell, C., Wong, T., \& Robertson, S. 2005, PASA, 22, 62

Lebouteiller, V., Bernard-Salas, J., Sloan, G. C., \& Barry, D. J. 2010, PASP, 122, 231

Lebouteiller, V., Cormier, D., Madden, S. C., et al. 2012, A\&A, 548, A91

Lee, J. C., Gil de Paz, A., Kennicutt, Jr., R. C., et al. 2011, ApJS, 192, 6

Leon, S., Combes, F., \& Menon, T. K. 1998, A\&A, 330, 37

Leroy, A., Bolatto, A. D., Simon, J. D., \& Blitz, L. 2005, ApJ, 625, 763

Leroy, A. K., Walter, F., Brinks, E., et al. 2008, AJ, 136, 2782

Leroy, A. K., Walter, F., Bigiel, F., et al. 2009, AJ, 137, 4670

Leroy, A. K., Bolatto, A., Gordon, K., et al. 2011, ApJ, 737, 12

MacHattie, J. A., Irwin, J. A., Madden, S. C., Cormier, D., \& Rémy-Ruyer, A. 2014, MNRAS, 438, L66

Madden, S. C. 2000, New Astron. Rev., 44, 249

Madden, S. C., Poglitsch, A., Geis, N., Stacey, G. J., \& Townes, C. H. 1997, ApJ, 483, 200

Madden, S. C., Rémy-Ruyer, A., Galametz, M., et al. 2013, PASP, 125, 600

Markarian, B. E., Lipovetsky, V. A., Stepanian, J. A., Erastova, L. K., \& Shapovalova, A. I. 1989, Soobshcheniya Spetsial'noj Astrofizicheskoj Observatorii, 62, 5

Marlowe, A. T., Meurer, G. R., Heckman, T. M., \& Schommer, R. 1997, ApJS, 112,285

Meier, D. S., Turner, J. L., Crosthwaite, L. P., \& Beck, S. C. 2001, AJ, 121, 740

Meier, D. S., Turner, J. L., \& Beck, S. C. 2002, AJ, 124, 877

Murphy, E. J., Condon, J. J., Schinnerer, E., et al. 2011, ApJ, 737, 67

Östlin, G., Amram, P., Masegosa, J., Bergvall, N., \& Boulesteix, J. 1999, A\&AS, 137,419

Östlin, G., Amram, P., Bergvall, N., et al. 2001, A\&A, 374, 800

Ott, S. 2010, in Astronomical Data Analysis Software and Systems XIX, eds.

Y. Mizumoto, K.-I. Morita, \& M. Ohishi, ASP Conf. Ser., 434, 139

Pérez-Montero, E., Vílchez, J. M., Cedrés, B., et al. 2011, A\&A, 532, A141

Pilbratt, G. L., Riedinger, J. R., Passvogel, T., et al. 2010, A\&A, 518, L1

Poglitsch, A., Krabbe, A., Madden, S. C., et al. 1995, ApJ, 454, 293

Poglitsch, A., Waelkens, C., Geis, N., et al. 2010, A\&A, 518, L2

Rémy-Ruyer, A., Madden, S. C., Galliano, F., et al. 2013, A\&A, 557, A95

Rémy-Ruyer, A., Madden, S. C., Galliano, F., et al. 2014, A\&A, 563, A31

Röllig, M., Ossenkopf, V., Jeyakumar, S., Stutzki, J., \& Sternberg, A. 2006,

A\&A, 451, 917

Roussel, H., Helou, G., Hollenbach, D. J., et al. 2007, ApJ, 669, 959

Rubin, V. C., Hunter, D. A., \& Ford, Jr., W. K. 1990, ApJ, 365, 86

Sage, L. J., Salzer, J. J., Loose, H.-H., \& Henkel, C. 1992, A\&A, 265, 19

Sandberg, A., Östlin, G., Hayes, M., et al. 2013, A\&A, 552, A95

Sanders, D. B., Scoville, N. Z., \& Soifer, B. T. 1991, ApJ, 370, 158

Sandstrom, K. M., Leroy, A. K., Walter, F., et al. 2013, ApJ, 777, 5

Schmidt, M. 1959, ApJ, 129, 243

Schmitt, H. R., Calzetti, D., Armus, L., et al. 2006, ApJS, 164, 52 
D. Cormier et al.: Molecular gas in dwarf galaxies

Schruba, A., Leroy, A. K., Walter, F., et al. 2012, AJ, 143, 138

Shetty, R., Glover, S. C., Dullemond, C. P., \& Klessen, R. S. 2011, MNRAS, 412,1686

Skillman, E. D., Côté, S., \& Miller, B. W. 2003, AJ, 125, 593

Smoker, J. V., Davies, R. D., Axon, D. J., \& Hummel, E. 2000, A\&A, 361, 19

Solomon, P. M., \& Sage, L. J. 1988, ApJ, 334, 613

Stacey, G. J., Geis, N., Genzel, R., et al. 1991, ApJ, 373, 423

Stacey, G. J., Hailey-Dunsheath, S., Ferkinhoff, C., et al. 2010, ApJ, 724, 957

Tacconi, L. J., \& Young, J. S. 1987, ApJ, 322, 681

Taylor, C. L., Kobulnicky, H. A., \& Skillman, E. D. 1998, AJ, 116, 2746

Terlevich, R., Melnick, J., Masegosa, J., Moles, M., \& Copetti, M. V. F. 1991, A\&AS, 91, 285

Thuan, T. X., \& Izotov, Y. I. 2005, ApJS, 161, 240

Thuan, T. X., Lipovetsky, V. A., Martin, J.-M., \& Pustilnik, S. A. 1999, A\&AS, 139,1
Tielens, A. G. G. M., \& Hollenbach, D. 1985, ApJ, 291, 722

van der Tak, F. F. S., Black, J. H., Schöier, F. L., Jansen, D. J., \& Van Dishoeck, E. F. 2007, A\&A, 468, 627

van Eymeren, J., Marcelin, M., Koribalski, B. S., et al. 2009, A\&A, 505, 105

Warren, B. E., Wilson, C. D., Israel, F. P., et al. 2010, ApJ, 714, 571

Werner, M. W., Roellig, T. L., Low, F. J., et al. 2004, ApJS, 154, 1

Wilcots, E. M., Lehman, C., \& Miller, B. 1996, AJ, 111, 1575

Williams, B. A., McMahon, P. M., \& van Gorkom, J. H. 1991, AJ, 101, 1957

Wilson, C. D., Howe, J. E., \& Balogh, M. L. 1999, ApJ, 517, 174

Wilson, C. D., Warren, B. E., Israel, F. P., et al. 2009, ApJ, 693, 1736

Wolfire, M. G., Hollenbach, D., McKee, C. F., Tielens, A. G. G. M., \& Bakes,

E. L. O. 1995, ApJ, 443, 152

Wolfire, M. G., Hollenbach, D., \& McKee, C. F. 2010, ApJ, 716, 1191

Wong, T., Hughes, A., Ott, J., et al. 2011, ApJS, 197, 16

Zubko, V., Dwek, E., \& Arendt, R. G. 2004, ApJS, 152, 211 